\title{
Physical Data of Soil Profiles Formed on Late Quaternary Marine Terraces near Santa Cruz, California
}

By Jennie Munster ${ }^{1}$ and Jennifer W. Harden ${ }^{1}$

${ }^{1}$ U.S. Geological Survey, 345 Middlefield Rd. MS 962, Menlo Park, CA 94025.

Table of Contents

$1.0 \quad$ Introduction

$2.0 \quad$ Methods

2.1 Field and bulk density

$2.2 \quad$ Particle size

2.3 Magnetic fraction

3.0 Descriptions of figures and tables (in parenthesis is the name of the downloadable file)

Figure 1 Location of terrace sites

Table 1 GPS of terrace sites

Table 2 Field descriptions

3.5

Table 3 Bulk density

3.6

Table 4 Particle size

3.7

Table 5 Magnetic fraction

3.8

Table 6 Soil auger descriptions

Table 7 Soil Development Index

4.0

References

5.0

Acknowledgments 


\subsection{Introduction}

The marine terraces in and around Santa Cruz, California represent a set of wellpreserved terraces formed as a product of geology, sea level and climate. A marine terrace begins as a wave cut platform. Eustatic sea level changes, sea cliff erosion and tectonic uplift work together to generate marine terraces. "When a wave-cut platform is raised (due to tectonic activity) above sea level and cliffed by wave action it becomes a marine terrace" (Bradley, 1957, pp.424). During glacial periods, eustatic sea level is estimated to have dropped by 150 meters (Fairbanks 1989). Cliff retreat measured from aerial photographs between 1930 and 1980 vary from 0.0 to $0.2 \mathrm{~m} \mathrm{y}^{-1}$ (Best and Griggs 1991). Estimates of uplift rates along the Santa Cruz coastline vary from 0.10 to $0.48 \mathrm{~m}$ kyr $^{-1}$ (Bradley and Griggs 1976, Weber and others 1999). Uplift mechanisms include coseismic uplift associated both with a reverse component of slip on the steeply SW dipping Loma Prieta fault in the restraining bend of the San Andreas Fault and a small component of reverse slip on the steeply SE dipping San Gregorio fault (Anderson and Menking 1994). Previous work studying physical properties on these terraces include Pinney and others (in press) and Aniku (1986) and Bowman and Estrada (1980).

Sedimentary deposits of the marine terraces are a mixture of terrestrial and marine sediments but generally consist of a sheet of marine deposits overlying the old platform and a wedge of non marine deposits banked against the old sea cliff (Bradley, 1957). Bedrock underlying the terraces in the Santa Cruz area is generally either Santa Margarita Sandstone or Santa Cruz Mudstone. The Santa Margarita Sandstone represents an upper Miocene, transgressive, tidally dominated marine-shelf deposit with crossbedded sets of sand and gravel and horizontally stratified and bioturbated invertebrate-fossils beds (Phillips, 1990). The siliceous Santa Cruz Mudstone, of late Miocene age, conformably overlies the Santa Margarita Sandstone. The Santa Cruz Mudstone is a thin to mediumbedded siliceous mudstone with nonsiliceous mudstone and siltstone and minor amounts of sandstone. The siliceous nature implies organic deposition in a quiescent, deep-water environment. Bedrock is mantled by 1-4 meters of medium to coarse-grained regressive beach sediment and fluvial deposits from the Ben Lomond Mountains.

Terrace age increases with elevation above sea level and weathering of primary minerals increases with age. The suite of soils formed on the terraces is referred to as a soil chronosequence. Soil chronosequences, important tools in characterizing natural 
weathering rates, are defined as a group of soils that differ in age and therefore in duration of weathering but have similar climatic conditions, vegetation, geomorphic position and parent material (Jenny, 1941; Birkland, 1999). Soils are frequently useful indicators of geomorphic age (Muhs, 1982; Switzer and others, 1988) and are a function of pedogenic and/or eolian processes. Some aspects of soil development can be episodic but when viewed on large time scales can be perceived as continuous (Switzer and others, 1988).

The age of the soil may be constrained by the age of the deposit, since soil formation generally commences when deposition has ceased (Birkland, 1999). Dating of the terraces provides an unprecedented opportunity to study weathering and soilformation rates (Perg and others, 2001; Hanks and others, 1984; Bradley and Griggs, 1976; Bradley and Addicott, 1968; Bradley, 1956). Ages of the terraces recently dated by cosmogenic radionuclide are, starting with the youngest, 65, 92, 137, 139 and $226 \mathrm{ky}$ (Perg and others, 2001). However these ages are much younger than recent radiometric dates on mollusk shells (Muhs, US Geological survey, personal communication 2002; Bradley and Addicott, 1968).

For this study soils were sampled on five terraces. Terrace one in the Lighthouse Field along Westcliff in Santa Cruz was the last site selected, and this report contains minimal data on this terrace. Sites on the second, third and fourth terraces are located in Wilder Ranch, Santa Cruz, California. Site five is on private property north of Wilder Ranch. Careful consideration was taken in selecting field sites, choosing locations in a topographically flat area to avoid effects of erosion and trying to keep parent material similar.

This report contains physical properties of the soil profiles on four of the five marine terraces near Santa Cruz, California, excluding the youngest terrace in all tables except 6 and 7. Data includes field descriptions, bulk density, grain size analyses, weight percent magnetic fraction and the soil development index. Soil properties are important when trying to understand the chemistry of a given profile or when comparing profiles. Grain size constrains the movement of water in a profile thus controlling movement of chemicals and weathering rates. Bulk density is a useful property to calculate chemical inventory. Quantifying the magnetic fraction aids in understanding the Fe inventory for these soils. The soil development index is a semi-quantitative way to define the degree of 
development of a soil profile. This is a useful way to compare development of profiles for this chronosequence or compare the Santa Cruz terraces to a suit of other terraces or another chronosequence.

\subsection{Methods}

2.1 Field and bulk density sampling

Samples were described in the field by J.W. Harden and J. Munster from hand dug soil pits according to the Soil Survey Manual. Other profiles were described from hand auger samples, by J. Munster according to the Soil Survey Manual and reported in Table 6.

Bulk density samples were extracted horizontally and vertically using cores of known diameter. The most consistent and accurate bulk density estimates for mineral soils were made from cores obtained with a Model 0200 coring device from Soilmoisture Equipment Corporation (Goleta, CA) in which internal rings can be disassembled to obtain intact samples. Samples were then placed on open shelves in an isolated room and allowed to air dry to a constant weight. After air-drying, samples were oven-dried for 48 hours in a forced-draft oven at $105^{\circ} \mathrm{C}$.

\subsection{Particle size}

Samples were acquired from splits of hand augured samples that had been oven dried and had particles $>2 \mathrm{~mm}$ removed. Since soil pit samples were not available at time of analysis samples were chosen using field notes to represent each horizon at each of the four terraces, excluding the youngest terrace. Later comparison with soil pits showed that not all horizons were represented.

Grain-size data was determined using two methods, sedigraph for particle size $<0.05 \mathrm{~mm}$ and dry sieving for particle size $>0.05 \mathrm{~mm}$. Classification of size fractions are according to the United States Department of Agriculture (Soil Survey Staff, 1998) and defined to be 2-1 $\mathrm{mm}$ as very coarse sand, 1-0.6 $\mathrm{mm}$ as coarse sand, 0.6-0.2 $\mathrm{mm}$ as medium sand, 0.2-0.1 $\mathrm{mm}$ as fine sand, 0.1-0.05 $\mathrm{mm}$ as very fine sand, 0.05-0.002 $\mathrm{mm}$ as silt and $<2 \mu \mathrm{m}$ as clay. Clay was then sub divided into $2-1 \mu \mathrm{m}, 1-0.5 \mu \mathrm{m}$ and $<0.5 \mu \mathrm{m}$ fractions. 
Preparation of samples included removing organic material by adding $\mathrm{H}_{2} \mathrm{O}_{2}$ and heating (no more than 1 hour) on a hot plate to evaporate the $\mathrm{H}_{2} \mathrm{O}_{2}$. Samples were deflocculated by adding a 5\% Calgon solution and put into an ultrasonic bath for five minutes. Wet sieving using a $0.05 \mathrm{~mm}$ in diameter sieve achieved separation of sand from clay/silt portions.

Dry sieving is a straightforward method, used for sands, where sieves are stacked with the largest size opening at the top. The sieves were agitated by mechanical means for three minutes. After sieving was completed the weight of the soil particles retained in each sieve is determined relative to the total sample weight and multiplied by 100 to obtain percentage.

Grain size distribution of silt and clays was determined utilizing a sedigraph. The sedigraph is utilized for its quickness of analysis. Using Stokes's Law and an X-Ray beam, the sedigraph monitors the rate at which particles settle and are removed from the monitored volume. Results yielded were in table form presenting mass \% finer vs. grain size. An analysis needs $\sim 50 \mathrm{~mL}$ of solution, solution volume is determined by concentration of particles in sample, and runs from $60 \mu \mathrm{m}$ to $0.5 \mu \mathrm{m}$. The mass $\%$ finer is applied directly to the amount of clay/silt fraction of the sample. Three samples were rerun to yield a standard deviation for the sedigraph method ( $<0.05 \mathrm{~mm}$ fraction) of $1.6 \%$.

\subsection{Magnetic Fraction}

Magnetic fraction was analyzed on splits from soil pit samples. These samples were gently crushed to pass through a $2 \mathrm{~mm}$ sieve, insuring no nodules or rocks were crushed.

Percent of magnetic fraction of $>2 \mathrm{~mm}$ particles was calculated as weight of magnetic particles $>2 \mathrm{~mm}$ divided by weight of all $>2 \mathrm{~mm}$ particles of the whole soil. The $>2 \mathrm{~mm}$ particles of the whole soil was weighed, a metal household magnet was thoroughly stirred throughout these particles, any particle attached to the magnet was transferred into another weighing dish until no particles were found to stick to the magnet. Those particles attached to the magnet were then weighed. The magnetic fraction could be underestimated if particles were too large for the magnet to move or if the particle was coated with a non-magnetic material, such as soil, to a point that magnetic material in the core of the particle was not affected by the magnet. 
Up to 5 grams of $<2 \mathrm{~mm}$ soil was ground to pass an 80 -mesh sieve, weighed, recorded to the $4^{\text {th }}$ decimal place, and then transferred into a crucible. A magnetic stirring bar was stirred throughout the soil to insure close contact with the soil and gently tapped against the side of the crucible to knock off any soil particles. The magnetic particles were removed from the magnetic stirring bar onto a clean glass plate. The glass plate was placed onto a magnetic stirrer and the stirrer was turned on and slowly increased to a medium setting. The material then began to migrate towards a spot on the center of the stirrer while separating from the soil. When all the soil was separated, the magnetic materials were weighed and the percentage calculated (Singer and others, 1986). A standard deviation of $0.0291 \%$ was calculated, from replicate separations of the same sample.

\subsection{Description of Figures and Tables}

3.1 Figure 1 Location of terrace sites

Locations for four terrace sites in Santa Cruz.

3.2 Table 1 GPS of terrace sites

Coordinates in UTM and Latitude/Longitude. Courtesy of David Stonestrom.

Location-Terrace ID and location of GPS measurements.

$\mathrm{N}$ data-Number of measurements recorded

StdErr XY-Error of measurements from the mean in meters

\subsection{Table 2 Field descriptions}

Profile ID-Used to identify the sample.

Basal Depth-Bottom depth of the horizon sampled in $\mathrm{cm}$.

Thickness-Horizon thickness in $\mathrm{cm}$.

Horizon ID-Horizon description according to conventions of the Soil Survey Staff (1998).

Sample Description-Brief field description of the horizon.

$\mathrm{pH}-$ The $\mathrm{pH}$ of the sample as defined in the field or in the lab using the LaMotte $\mathrm{pH}$ field kit.

Munsell Moist Color-Soil color according to the Munsell Color chart, when only dry color was described a D is used to indicate this.

Roots-Root abundance and size according to conventions of Soil Survey Staff (1998). 
Structure-Soil structure abundance, size and type according to conventions of Soil Survey Staff (1998).

Stickiness-Stickiness according to conventions of Soil Survey Staff (1998).

Plasticity—Plasticity according to conventions of Soil Survey Staff (1998).

Moist or Dry Consistence-Consistence, depending on if soil was moist or dry descriptors was used according to conventions of Soil Survey Staff (1998).

Texture-Soil texture as determined in the field according to conventions of Soil Survey Staff (1998).

\subsection{Table 3 Bulk density}

Profile ID—Used to identify the sample.

Basal Depth-Bottom depth of the horizon sampled in $\mathrm{cm}$.

Thickness-Horizon thickness in $\mathrm{cm}$.

Horizon ID-Horizon description according to conventions of the Soil Survey Staff (1998).

Sample Description-Brief field description of the horizon.

Average Bulk Density AD—An average of 1-3 samples calculating grams of air-dry soil per cubic centimeter

Average Bulk Density OD-An average of 1-3 samples calculating grams of oven-dry soil per cubic centimeter

Notes-Indicates how many samples and depth of samples that were used in averaging bulk density values.

\subsection{Table 4 Particle size}

Profile ID-Used to identify the sample.

Basal Depth-Bottom depth of the horizon sampled in $\mathrm{cm}$.

Horizon ID-Horizon description according to conventions of the Soil Survey Staff (1998).

Texture- Soil texture as defined by particle size distribution according to conventions of Soil Survey Staff (1998).

All particle size distributions are defined by USDA classifications (see section 3.2).

\subsection{Table 5 Magnetic fractions}

Profile ID—Used to identify the sample.

Basal Depth—Bottom depth of the horizon sampled in $\mathrm{cm}$. 
Horizon ID-Horizon description according to conventions of the Soil Survey Staff (1998).

Sample Description—Brief field description of the horizon.

$>2 \mathrm{~mm}$ Fraction-Percent of $>2 \mathrm{~mm}$ fraction that is magnetic.

$<2 \mathrm{~mm}$ Average Fraction-Calculated average percent of $<2 \mathrm{~mm}$ fraction that is magnetic.

$<2 \mathrm{~mm} \mathrm{SD}(\mathrm{n}=2)-$ Calculated standard deviation for two runs on the same sample. Below the sample standard deviations is the average standard deviation for all samples.

A “-“ indicates no analysis performed

3.7 Soil auger descriptions

Terrace-Terrace location.

Sample ID—Used to identify the sample.

Basal Depth-Bottom depth of horizon.

Description Interval-The depths at which the sample was described

Sample Description-Brief description of horizon.

Roots Present—Described according to the size distributions of Soil Survey Staff (1998), abundance could not be determined in augur samples.

Dry Munsell Color-Soil color according to the Munsell Color chat.

Texture-Soil texture as determined in the field according to conventions of Soil Survey Staff (1998).

Stickiness-Stickiness according to conventions of Soil Survey Staff (1998).

Plasticity—Plasticity according to conventions of Soil Survey Staff (1998).

3.8 Soil development index

Soil properties were quantified according to Harden (1982) and Harden and Taylor (1983) and an index was used to combine three to four soil field properties. These properties are: texture (combination of texture and wet consistence), rubification (color hue and chroma), melanization (color value) and $\mathrm{pH}$ lowering (only samples from hand dug soil pits have $\mathrm{pH}$ values).

A sample from Wilder Beach was used as the parent material for all soils and all horizons. While the terraces are likely a mix of marine and fluvial sediments, the sediment source of the beach is likely similar to sources of sediment during terrace 
formation as the bedrock is similar throughout the study area. Wilder Beach sand has a dry Munsell color of 10YR7/2, texture of sand-non sticky, non plastic- and a pH of 7.2.

To normalize data, maximum values are used according to Harden (1982). These values for texture, rubification, melanization, and $\mathrm{pH}$ are $90,190,70$ and 3.5 respectively. However due to the advanced development of many horizons, total texture values exceeded this maximum yielding values ranging between 0-1.3. A value greater than one shows that (1) these horizons are more developed than soils near Merced (Harden, 1982) or (2) parent material for these horizons may be misrepresented.

Most values of rubification and melanization use only dry color values. When a horizon has only a dry or a wet value the quantity is multiplied by two, in few cases the dry and wet values are summed.

This table presents quantified soil field properties, normalized data, sum of normalized properties, horizon index (n), index-cm of development for all horizons and index-cm and average index/cm for each profile.

\subsection{References}

Anderson, R.S., and Menking, K.M., 1994, The Quaternary marine terraces of Santa Cruz, California; evidence for coseismic uplift on two faults: Geological Society of America Bulletin, v.106, no.5, p. 649-664.

Aniku, J.R.F., 1986, Trends of pedogenic iron oxides in a marine terrace chronosequence: PhD dissertation, University of California, Davis, p. 227.

Best, T.C., and Griggs, G.B., 1991, A sediment budget for the Santa Cruz littoral cell: California Society of Economic Paleontologists and Mineralogists, v.46, p. 35-50.

Birkland, P.W., 1999, Soils and geomorphology: Oxford University Press, New York, United States, p. 430.

Bownam, R.H., and Estrada, D.C., 1980, Soil Survey of Santa Cruz County, California. U.S. Dep. Agric., Soil Conserv. Serv., Washington, DC, United States, p. 148.

Bradley, W., 1956, Carbon-14 date for a marine terrace at Santa Cruz, California:

Geological Society of America Bulletin, v.67, no. 5, p. 675-677.

Bradley, W., 1957 Origin of marine-terrace deposits in the Santa Cruz area, California: Geological Society of America Bulletin, v.68, no. 4, p. 421-444. 
Bradley, W., and Addicott, W., 1968, Age of first marine terrace near Santa Cruz, California: Geological Society of America Bulletin, v.79, no.9, p. 1203-1209.

Bradley, W.C., and Griggs, G.B., 1976, Form, genesis, and deformation of central California wave-cut platforms: Geological Society of America Bulletin, v.87, no.3, p. 433-449.

Fairbanks, R.G., 1989, A 17,000-year glacio-eustatic sea level record; influence of glacial melting rates on the Younger Dryas event and deep-ocean circulation: Nature (London), v.342, no.6250, p.637-642.

Hanks, T.C., Bucknam R.C., Lajoie K.R., and Wallace, R.E., 1984, Modification of wave-cut and faulting-controlled landforms: Journal of Geophysical Research B v.89, no.7, p.5771-5790.

Harden, J.W., 1982, A quantitative index of soil development from field descriptions: examples from a chronosequence in Central California: Geoderma v.28, p 1-28.

Harden, J.W., and Taylor, E.M., 1983, A quantitative comparison of soil development in four climatic regimes: Quaternary Research v. 20, p. 3452-349.

Jenny, H., 1941, Factors of soil formation: New York, McGraw-Hill Book Co, p. 281.

Muhs, D.R., 1982, A soil chronosequence on quaternary marine terraces, San Clemente Island, California: Geoderma v.28, p. 257-283.

Pinney, C., Aniku, J., Burke, R., Harden, J.W., Singer, M, and. Munster J., in press, Soil chemistry and mineralogy of the Santa Cruz Coastal Terraces: US Geological Survey Open File Report

Perg, L.A., Anderson, R.A., and Finkel, R.C., 2001a, Use of a new ${ }^{10} \mathrm{Be}$ and ${ }^{26} \mathrm{Al}$ inventory method to date marine terraces, Santa Cruz, California, USA: Geology, v.29, no. 10 , p. 879-882.

-- $\quad 2001 \mathrm{~b}$, Cosmogenic radionuclide constraints on active margin coastline uplift and geomorphic rates, Santa Cruz, California, USA: PhD dissertation, University of Californiz, Santa Cruz.

Phillips, L., 1990, Depositional and structural controls on the distribution of tar sands in the Santa Cruz Mountains, California: Guidebook - Pacific Section, American Association of Petroleum Geologists, v.67, p.105-121.

Singer, M..J., and Janitzky, P., Editors, 1986, Field and Laboratory Procedures Used in a Soil Chronosequence Study: U.S. Geological Survey Bulletin 1648. 
Soil Survey Staff, 1998. Keys to Soil Taxonomy. U.S. Dept. Agric., $8^{\text {th }}$ ed, Washington D.C., p. 326.

Soil Survey Staff, Soil Taxonomy, 1975, A basic system of soil classification for making and interpreting soil surveys: Agriculture Handbook no.436 . Soil Conservation Service U.S. Department of Agriculture.

Switzer, P., Harden, J.W., and Mark, R.K., 1998, A statistical method for estimating rates of soil development and ages of geologic deposits; a design for soilchronosequence studies: Mathematical Geology, v.20, no.1, p. 49-61.

Weber, G.E., Nolan, J.M., and Zinn, E.N., 1999, Late Quaternary slip across the San Gregorio Fault Zone, San Mateo County, California: Estimates from marine terrace offsets: AAPG Bulletin v.83, no.4 p. 705.

\subsection{Acknowledgements}

The author would like to thank Dr. Slawek Tulaczyk at the University of Santa Cruz California for supervising the particle size work done there as a senior thesis. She would like to thank Art White and Marjorie Schulz at the U.S.G.S. for their careful consideration and effort in deliberating where sample sites were to go and for acquiring samples for particle size analysis. She would also like to thank Jennifer Harden at the U.S.G.S. for her assistance in describing the soil pits and her helpful comments in my process learning more about the soil development index. She also wants to thank the other many members in the field crew that helped in manual labor, such as digging the soil pits and taking field notes. 


\section{Map of Terrace Locations}

fig.1a

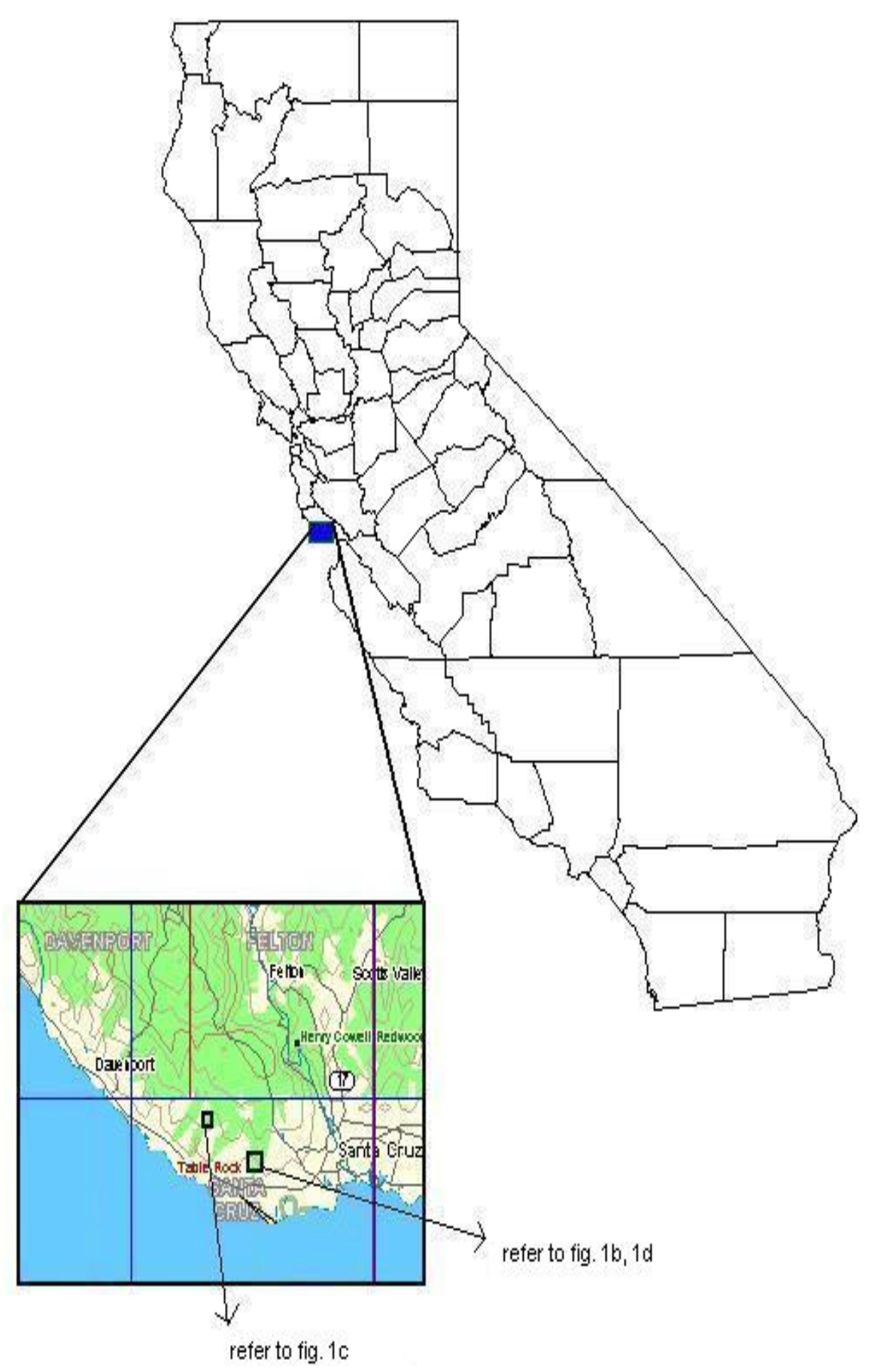

1 of 2 


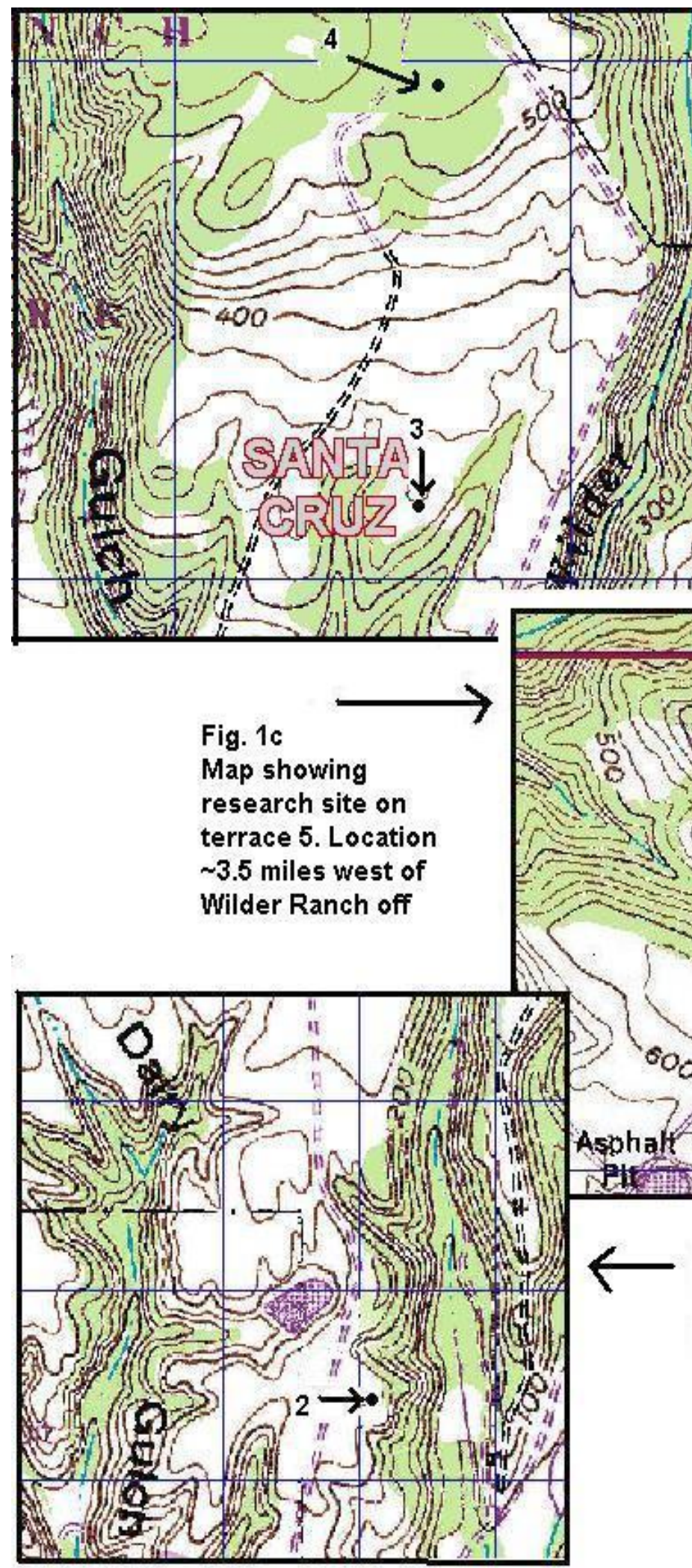

Fig. 1b

Map of research sites on

terrace 3 and 4 . Located

inside Wilder Ranch,

Santa Cruz, California

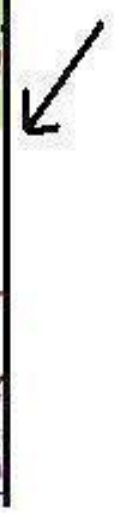

Fig. 1c

Map showing

research site on

terrace 5 . Location

$\sim 3.5$ miles west of

Wilder Ranch off

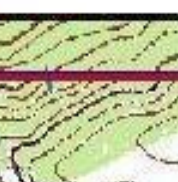

Sprixth 
SANTA CRUZ GPS LOCATIONS (DATUM = NAD27 CONUS): ACCUMULATIVE AVERAGE POSITIONS:

\begin{tabular}{|c|c|c|c|c|c|c|c|}
\hline Location & $\begin{array}{l}\text { UTM } \\
\text { Zone }\end{array}$ & $\begin{array}{l}\text { Easting } \\
\text { (m) }\end{array}$ & $\begin{array}{l}\text { Northing } \\
\text { (m) }\end{array}$ & $\mathbf{N}$ data & $\begin{array}{l}\text { StdErr XY } \\
\text { (m) }\end{array}$ & N Latitude & W Longitude \\
\hline SC-T2 & $10 \mathrm{~S}$ & 581446 & 4091507 & 4 & 2.5 & $36^{\circ} 58^{\prime} 04.69^{\prime \prime}$ & $122^{\circ} 05^{\prime} 06.14^{\prime \prime}$ \\
\hline SC-T3 & $10 \mathrm{~S}$ & 582097 & 4092444 & 3 & 3.1 & $36^{\circ} 58^{\prime} 34.90^{\prime \prime}$ & $122^{\circ} 04^{\prime} 39.47^{\prime \prime}$ \\
\hline SC-T4 & $10 \mathrm{~S}$ & 582143 & 4093249 & 3 & 1.3 & $36^{\circ} 59^{\prime} 00.98^{\prime \prime}$ & $122^{\circ} 04^{\prime} 37.30^{\prime \prime}$ \\
\hline SC-T5 & $10 \mathrm{~S}$ & 577144 & 4094428 & 3 & 1.6 & $36^{\circ} 59^{\prime} 40.78^{\prime \prime}$ & $122^{\circ} 08^{\prime} 00.00^{\prime \prime}$ \\
\hline
\end{tabular}




\section{Basal Thick - Horizo}

ID

\section{$\begin{array}{cc}(\mathrm{cm}) & (\mathrm{cm})\end{array}$}

SCT2 1.5

SCT2 1.39

SCT2 1.50

SCT2 1.68

SCT2 1.88

SCT2 2.12

SCT2 2.22

SCT2 2.65

SCT2 2.85

SCT2 2.100

SCT3 1.5
SCT 31.10

SCT3 1.20

SCT3 1.30

SCT3 1.5

SCT3 1.70

$\begin{array}{lll}\text { SCT3 } & 1.90 \\ \text { SCT3 } & 1.100\end{array}$

SCT4 1.10

SCT4 1.20
SCT4 1.28

SCT4 1.40

SCT4 1.80

SCT4 1.100

SCT5 1.10

SCT5 1.20

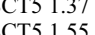

SCT5 1.80

SCT5 1.100
Sample Description

Darkened topsoil with many roots

Dark topsoil with roots

Dark, plastic subsoil with roo

Dark, plastic subsoil with roots

B Glated gay sibsil with roos

Glayed, gray subsoil with roots

Topsoil rooting zone

AB Horizon with topsoil and mineral mixing

Mineral horizon with Fe concretions

Mineral horizon with Fe stains, nodules and redox features

Mineral horizon with redoxy morphic features

\section{Dark topsoil with many roots}

Dark topsoil with many roots

B Dark subsoil w/ nodules from above and roots

B Dark subsoil $\mathrm{w} /$ nodules from below and roots

Mottled subsoil with feldsbar, clay films and roots

Dark subsoil with few pebbles and roots

Highly mottled subsoil

\section{A Dark topsoil with roots}

Dark topsoil with roots

Dark topsoil with roots

Dark subsoil with clay films and roots

Dark subsoil with clay films and roots
Dark subsoil with mottling and roots on ped faces

Dark subsoil with mottling

A Dark topsoil with roots

AB Dark subsoil with roots

Dark subsoil w/red pebbles and $v$ fine roots along ped faces 5.6

Dark subsoil w/red pebbles and v.fine roots along ped faces 5.8

Dark subsoil with clay films, common red pebbles
Dark subsoil with common v.fine roots along ped faces

$\begin{array}{cc}\text { pH } & \begin{array}{c}\text { Munsell } \\ \text { Moist Color } \\ D=d r y\end{array} \\ & \\ 6.0 & 10 \mathrm{YR} 3 / 3 \\ 6.0 & 10 \mathrm{YR} 3 / 3 \\ 5.5 & 10 \mathrm{YR} 3 / 2 \\ 7.0 & 10 \mathrm{YR} 4 / 6 \\ 7.0 & 10 \mathrm{YR} 4 / 6 \\ 7.0 & 10 \mathrm{YR} 4 / 3 \\ 7.0 & 10 \mathrm{YR} 4 / 1 \\ & \\ - & 10 \mathrm{YR} 3 / 3 \\ - & 10 \mathrm{YR} 2 / 2 \\ - & 10 \mathrm{YR} 4 / 4 \\ - & 10 \mathrm{YR} 4 / 4 \\ - & 10 \mathrm{YR} 4 / 2 \\ - & 10 \mathrm{YR} 4 / 1 \\ & \\ 5.5 & 10 \mathrm{YR} 3 / 2 \\ 5.6 & 10 \mathrm{YR} 3 / 2 \\ 5.8 & 10 \mathrm{YR} 4 / 3 \\ 5.6 & 10 \mathrm{YR} 3 / 3 \\ 5.8 & 10 \mathrm{YR} 4 / 4 \\ 5.6 & 10 \mathrm{YR} 6 / 8 \mathrm{D} \\ 5.7 & 10 \mathrm{YR} 4 / 2 \mathrm{D} \\ 5.4 & 10 \mathrm{YR} 5 / 1 \mathrm{D} \\ & \\ 6.0 & 10 \mathrm{YR} 3 / 3 \\ 6.0 & 10 \mathrm{YR} 3 / 4 \\ 6.2 & 7.5 \mathrm{YR} 4 / 6 \\ 6.2 & 7.5 \mathrm{YR} 5 / 6 \mathrm{D} \\ 6.6 & 7.5 \mathrm{YR} 6 / 8 \mathrm{D} \\ 6.4 & 7.5 \mathrm{YR} 4 / 6 \\ 7.0 & 7.5 \mathrm{YR} 5 / 0 \\ & \\ 6.2 & 10 \mathrm{YR} 4 / 2 \\ 6.2 & 10 \mathrm{YR} 3 / 3 \\ 6.6 & 10 \mathrm{YR} 3 / 3 \\ 5.8 & 10 \mathrm{YR} 5 / 4 \\ 5.6 & 10 \mathrm{YR} 4 / 4 \\ 5.2 & 10 \mathrm{YR} 4 / 1 \mathrm{D} \\ & \end{array}$

Roots
common very fine, many fine
common very fine, many fine
few very fine and fine
few very fine
few very fine
few very fine
very few very fine

many very fine and fine common very fine, few fine

ew very fine and fine

not present

ew very fine

common v.fine, many fine common v.fine and fine v.few fine, common v.fine few very fine

few very fine and fine

few very fine

few fine, common very fine few fine and very fine few fine and very fin few very fine

few very fine and mediun few very fine

common fine

common fine and medium common $\mathrm{v}$ fine, fine and medi

$$
\begin{aligned}
& \text { few very fine } \\
& \text { few very fine }
\end{aligned}
$$

Structure

common medium granular common medium granular

few coarse subangular blocky

few v.coarse subangular blocky

ew v coarse subangular blocky

few medium subangular blocky

many coarse granular

common coarse subangluar blocky

many medium subangluar blocky

many coarse subangular blocky

many coarse subangular blocky

many coarse subangular blocky

many fine subangular blocky

common coarse subangular blocky

few coarse subangular blocky

many medium subangular blocky

common fine prismatic

many v.coarse subangular blocky

common coarse subangular blocky

common coarse subangular blocky

common v.coarse prismatic

common v.coarse prismatic

few v.coarse prismatice

many coarse subangular blocky

many fine-coarse subangular blocky

many medium subangular blocky

many coarse prismatic
Stickiness

Plasticity

Moist or Dry

Consistence

Texture

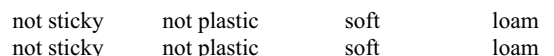
slightly sticky slightly plastic very friable loam slightly sticky plastic very friable silty clay loam slightly sticky plastic very friable silty clay loan plastic friable clay loam slightly sticky slightly plastic very friable loam $\begin{array}{llll} & \text { lightly sticky slightly plastic } & \text { friable } & \text { loam }\end{array}$ sticky plastic friable loam friable clay loam

slightly sticky slightly plastic slightly hard loam slightly sticky slightly plastic slightly hard loam slightly sticky slightly plastic slightly hard loam slightly sticky slightly plastic slightly hard sticky slightly plastic soft sticky plastic very hard sticky plastic very hard clay loam not sticky not plastic slightly hard silt loam slightly plastic slightly har sticky plastic slightly sticky slightly plastic lightly sticky slightly plastic sticky plastic

hard hard hard hard loam clay loam clay loam loam loam loam clay loam clay loam clay loam 
$(\mathrm{cm}) \quad(\mathrm{cm})$

SCT2 1.5

SCT2 1.13

SCT2 1.39

SCT2 1.50

SCT2 1.68

SCT2 1.88

SCT2 1.100

SCT3 1.5

SCT3 1.10

SCT3 1.20

SCT3 1.30

SCT3 1.55

SCT3 1.70

SCT3 1.90

SCT4 1.10

SCT4 1.20

SCT4 1.28

SCT4 1.40

SCT4 1.60

SCT4 1.80

SCT5 1.10

SCT5 1.20

SCT5 1.37

SCT5 1.55

SCT5 1.80

SCT5 1.100

\section{Sample Description}

Darkened topsoil with many roots

Darkened topsoil with many roots

Dark topsoil with roots

AB Dark, plastic subsoil with roots

AB Dark, plastic subsoil with roots

B Slightly mottled subsoil with roots

B Glayed, gray subsoil with roots

A Dark topsoil with many roots

A Dark topsoil with many roots

A Dark topsoil with many roots

AB Dark subsoil w/ nodules from above and roots

AB Dark subsoil $w /$ nodules from below and roots

B Mottled subsoil with feldsbar, clay films and roots

B Dark subsoil with few pebbles and roots

B Highly mottled subsoil

A Dark topsoil with roots

$\mathrm{AB}$ Dark topsoil with roots

AB Dark topsoil with roots

B Dark subsoil with clay films and roots

B Dark subsoil with clay films and roots

B Dark subsoil with mottling and roots on ped faces

B Dark subsoil with mottling

A Dark topsoil with roots

A Dark topsoil with roots

AB Dark subsoil with roots

B Dark subsoil w/ red pebbles and v.fine roots along ped faces

B Dark subsoil with clay films, common red pebbles

B Dark subsoil with common v.fine roots along ped faces

\begin{tabular}{|c|c|c|}
\hline $\begin{array}{c}\text { Average Bulk } \\
\text { Density AD } \\
\left(\mathrm{g} / \mathrm{cm}^{3}\right)\end{array}$ & $\begin{array}{c}\text { Average Bulk } \\
\text { Density OD } \\
\left(\mathrm{g} / \mathrm{cm}^{3}\right)\end{array}$ & Notes \\
\hline $\begin{array}{l}0.7947 \\
1.1142 \\
1.4037 \\
1.5838 \\
1.4885 \\
1.4641 \\
1.4864\end{array}$ & $\begin{array}{l}0.7846 \\
1.0968 \\
1.3868 \\
1.5649 \\
1.4613 \\
1.4318 \\
1.4450\end{array}$ & $\begin{array}{l}\text { Average of three samples taken. } \\
\text { Average of samples taken at } 8,7 \text {, and } 11 \mathrm{~cm} \text {. } \\
\text { Average of samples taken at } 27 \text { and } 34 \mathrm{~cm} \text {. } \\
\text { Average of samples taken at } 43 \text { and } 48 \mathrm{~cm} \text {. } \\
\text { Average of samples taken at } 54 \text { and } 59 \mathrm{~cm} \text {. } \\
\text { Average of samples taken at } 77 \text { and } 78 \mathrm{~cm} \text {. } \\
\text { Average of samples taken at } 90 \text { and } 91 \mathrm{~cm} \text {. }\end{array}$ \\
\hline $\begin{array}{c}0.9407 \\
1.4738 \\
1.2967 \\
1.3196 \\
1.3599 \\
1.3562 \\
1.3696 \\
\text { ND }\end{array}$ & $\begin{array}{c}0.9272 \\
1.4489 \\
1.2781 \\
1.3030 \\
1.3439 \\
1.3402 \\
1.3025 \\
\text { ND }\end{array}$ & $\begin{array}{l}\text { Average of two samples. } \\
\text { Sample taken at } 15 \mathrm{~cm} . \\
\text { Sample with base depth of } 30 \mathrm{~cm} . \\
\text { Sample taken at } 40 \mathrm{~cm} . \\
\text { Sample taken at } 60 \mathrm{~cm} . \\
\text { Sample taken at } 80 \mathrm{~cm} .\end{array}$ \\
\hline $\begin{array}{l}1.3835 \\
1.3937 \\
1.5431 \\
1.6309 \\
1.5809 \\
1.2021 \\
\text { ND }\end{array}$ & $\begin{array}{l}1.3662 \\
1.3791 \\
1.5282 \\
1.6141 \\
1.5447 \\
1.1586 \\
\text { ND }\end{array}$ & $\begin{array}{l}\text { Average of samples with base depths of } 5,7 \text {, and } 7 \mathrm{~cm} \text {. } \\
\text { Average of samples with base depths of } 18,18 \text {, and } 20 \mathrm{~cm} \\
\text { Average of samples with base depths of } 27 \text { and } 27 \mathrm{~cm} \text {. } \\
\text { Average of samples with base depths of } 31 \text { and } 39 \mathrm{~cm} \text {. } \\
\text { Average of samples with base depths of } 46,49 \text {, and } 53 \mathrm{~cm} \\
\text { Sample has base depth of } 62 \mathrm{~cm} \text {. }\end{array}$ \\
\hline $\begin{array}{l}\text { ND } \\
\text { ND } \\
\text { ND } \\
\text { ND } \\
\text { ND } \\
\text { ND }\end{array}$ & $\begin{array}{l}\text { ND } \\
\text { ND } \\
\text { ND } \\
\text { ND } \\
\text { ND } \\
\text { ND }\end{array}$ & \\
\hline
\end{tabular}




\begin{tabular}{|c|c|c|c|c|c|c|c|c|c|c|c|c|c|c|}
\hline Profile ID & $\begin{array}{c}\text { Basal } \\
\text { Depth } \\
(\mathrm{cm})\end{array}$ & Horizon & Texture & $\begin{array}{c}\text { Total } \\
\text { Sand } \\
(\%)\end{array}$ & $\begin{array}{c}\text { V. Coarse } \\
\text { Sand } \\
(\%)\end{array}$ & $\begin{array}{c}\text { Coarse } \\
\text { Sand } \\
(\%)\end{array}$ & $\begin{array}{c}\text { Medium } \\
\text { Sand } \\
(\%)\end{array}$ & $\begin{array}{c}\text { Fine } \\
\text { Sand } \\
(\%)\end{array}$ & $\begin{array}{c}\text { V. Fine } \\
\text { Sand } \\
(\%)\end{array}$ & $\begin{array}{l}\text { Silt } \\
(\%)\end{array}$ & $\begin{array}{c}\text { Total Clay } \\
<2 \mu \mathrm{m} \\
(\%)\end{array}$ & $\begin{array}{c}\text { Clay } \\
2-1 \mu \mathrm{m} \\
(\%)\end{array}$ & 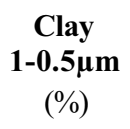 & $\begin{array}{c}\text { Clay } \\
<0.5 \mu \mathrm{m} \\
(\%)\end{array}$ \\
\hline SCT2-0.10 & 10 & A & sandy clay loam & 51.30 & 1.26 & 3.47 & 14.88 & 22.15 & 9.53 & 23.76 & 21.75 & 2.64 & 2.64 & 16.47 \\
\hline SCT2-0.22 & 22 & A & sandy clay loam & 52.29 & 1.60 & 3.14 & 14.27 & 22.70 & 10.58 & 20.88 & 18.81 & 2.18 & 2.42 & 14.21 \\
\hline SCT2-0.48 & 48 & $\mathrm{AB}$ & sandy clay loam & 50.66 & 1.27 & 2.66 & 14.02 & 23.22 & 9.49 & 22.89 & 18.28 & 4.08 & 6.51 & 7.70 \\
\hline SCT2-0.59 & 59 & $\mathrm{AB}$ & sandy clay loam & 55.05 & 1.34 & 2.85 & 16.42 & 24.84 & 9.60 & 21.00 & 22.75 & 2.84 & 11.42 & 8.49 \\
\hline SCT2-0.79 & 79 & B & sandy clay loam & 56.77 & 1.52 & 3.20 & 16.41 & 24.99 & 10.65 & 12.75 & 25.09 & 3.86 & 16.54 & 4.69 \\
\hline SCT2-0.95 & 95 & B & sandy clay & 53.59 & 1.45 & 3.14 & 11.54 & 22.52 & 14.93 & 9.47 & 28.72 & 1.83 & 1.87 & 25.02 \\
\hline SCT2-6.48 & 648 & & loamy sand & 84.17 & 4.88 & 5.67 & 49.70 & 18.90 & 5.02 & 5.05 & 2.31 & 0.68 & 0.35 & 1.29 \\
\hline SCT3-0.10 & 10 & $\mathrm{~A}$ & sandy clay loam & 52.01 & 0.99 & 2.10 & 14.10 & 21.49 & 13.33 & 25.38 & 21.88 & 4.25 & 4.49 & 13.14 \\
\hline SCT3-0.45 & 45 & $\mathrm{AB}$ & sandy clay loam & 50.73 & 2.66 & 2.42 & 13.81 & 21.40 & 10.44 & 20.83 & 28.42 & 2.07 & 2.51 & 23.84 \\
\hline SCT3-0.54 & 54 & $\mathrm{AB}$ & sandy clay loam & 54.38 & 2.26 & 2.02 & 14.24 & 23.87 & 11.99 & 25.41 & 19.80 & 3.48 & 5.61 & 10.72 \\
\hline SCT3-1.59 & 159 & $\mathrm{~B}$ & sandy clay loam & 63.12 & 1.75 & 3.15 & 21.37 & 30.94 & 5.91 & 10.44 & 26.19 & 2.82 & 3.33 & 20.03 \\
\hline SCT3-1.91 & 191 & $\mathrm{C}$ & sandy loam & 79.89 & 2.37 & 3.71 & 24.76 & 41.69 & 7.36 & 6.88 & 12.73 & 1.82 & 1.98 & 8.92 \\
\hline SCT3-7.31 & 731 & & sandy loam & 79.75 & 0.13 & 0.40 & 4.09 & 51.35 & 23.78 & 7.17 & 12.26 & 2.56 & 2.82 & 6.88 \\
\hline SCT4-0.01 & 1 & $\mathrm{~A}$ & sandy loam & 68.64 & 3.56 & 8.34 & 26.50 & 22.41 & 7.83 & 16.95 & 14.56 & 3.25 & 11.31 & 0.00 \\
\hline SCT4-0.33 & 33 & $\mathrm{AB}$ & sandy clay loam & 53.95 & 3.38 & 5.25 & 19.77 & 19.56 & 5.99 & 15.61 & 30.04 & 2.69 & 3.88 & 23.46 \\
\hline SCT4-0.50 & 50 & $\mathrm{~B}$ & clay & 32.86 & 2.16 & 3.69 & 11.95 & 11.53 & 3.53 & 9.18 & 57.35 & 1.86 & 2.99 & 52.49 \\
\hline SCT4-1.21 & 121 & B & sandy clay loam & 54.45 & 4.30 & 5.51 & 11.90 & 27.62 & 5.12 & 12.25 & 32.95 & 3.71 & 29.24 & 0.00 \\
\hline SCT4-3.96 & 396 & $\mathrm{C}$ & sandy loam & 76.93 & 0.44 & 1.43 & 16.11 & 33.86 & 25.09 & 10.89 & 11.61 & 2.11 & 9.47 & 0.02 \\
\hline SCT4-4.75 & 475 & & loamy sand & 83.69 & 0.55 & 0.85 & 21.48 & 43.55 & 17.27 & 7.98 & 7.82 & 2.16 & 1.86 & 3.79 \\
\hline SCT5-0.11 & 11 & A & sandy clay loam & 59.29 & 1.05 & 3.51 & 21.46 & 13.31 & 19.96 & 18.14 & 22.08 & 2.53 & 3.10 & 16.45 \\
\hline SCT5-0.33 & 33 & $\mathrm{AB}$ & sandy clay loam & 48.00 & 1.10 & 3.42 & 20.42 & 12.91 & 10.14 & 23.20 & 28.36 & 2.63 & 2.84 & 22.89 \\
\hline SCT5-0.54 & 54 & $\mathrm{~B}$ & clay & 30.18 & 0.69 & 2.23 & 13.66 & 8.58 & 5.02 & 13.60 & 56.15 & 2.44 & 3.91 & 49.80 \\
\hline SCT5-1.70 & 170 & $\mathrm{C}$ & clay & 40.58 & 0.60 & 4.21 & 20.78 & 10.59 & 4.40 & 8.24 & 51.03 & 1.24 & 1.48 & 48.31 \\
\hline SCT5-6.29 & 629 & & loamy sand & 79.36 & 0.49 & 1.51 & 13.23 & 56.57 & 7.56 & 12.11 & 8.28 & 1.96 & 1.39 & 4.93 \\
\hline
\end{tabular}




\begin{tabular}{|c|c|c|c|c|c|c|}
\hline Profile ID & $\begin{array}{c}\text { Basal } \\
\text { Depth } \\
(\mathrm{cm})\end{array}$ & $\begin{array}{c}\text { Horizon } \\
\text { ID }\end{array}$ & Sample Description & $\begin{array}{c}>2-\mathrm{mm} \\
\text { Fraction } \\
\quad(\%)\end{array}$ & $\begin{array}{c}<2-\mathrm{mm} \\
\text { Average } \\
\text { Fraction } \\
(\%)\end{array}$ & $\begin{array}{c}<2-\mathbf{m m} \\
\text { SD }(\mathrm{n}=\mathbf{2}) \\
(\%)\end{array}$ \\
\hline SCT2 1.5 & 5 & A & Darkened topsoil with many roots & 21.43 & 0.1705 & - \\
\hline SCT2 1.13 & 13 & A & Darkened topsoil with many roots & 40.00 & 0.0753 & - \\
\hline SCT2 1.39 & 39 & A & Dark topsoil with roots & 43.75 & 0.0289 & 0.0006 \\
\hline SCT2 1.50 & 50 & $\mathrm{AB}$ & Dark, plastic subsoil with roots & 45.28 & 0.0248 & - \\
\hline SCT2 1.68 & 68 & $\mathrm{AB}$ & Dark, plastic subsoil with roots & 23.08 & 0.0266 & - \\
\hline SCT2 1.88 & 88 & $\mathrm{~B}$ & Slightly mottled subsoil with roots & 0.00 & 0.0139 & 0.0091 \\
\hline SCT2 1.100 & 100 & $\mathrm{~B}$ & Glayed, gray subsoil with roots & 0.00 & 0.0075 & 0.0006 \\
\hline SCT2 2.12 & 12 & $\mathrm{~A}$ & Topsoil rooting zone & - & - & - \\
\hline SCT2 2.22 & 22 & $\mathrm{~A}$ & Topsoil rooting zone & - & - & - \\
\hline SCT2 2.44 & 44 & $\mathrm{AB}$ & Horizon with topsoil and mineral mixing & - & - & - \\
\hline SCT2 2.65 & 65 & $\mathrm{~B}$ & Mineral horizon with Fe concretions & - & - & - \\
\hline SCT2 2.85 & 85 & $\mathrm{~B}$ & Mineral horizon with Fe stains, nodules and redox features & - & - & - \\
\hline SCT2 2.100 & 100 & $\mathrm{~B}$ & Mineral horizon with redoxy morphic features & - & - & - \\
\hline SCT3 1.5 & 5 & A & Dark topsoil with many roots & 24.18 & 0.0635 & - \\
\hline SCT3 1.10 & 10 & A & Dark topsoil with many roots & 19.97 & 0.0347 & - \\
\hline SCT3 1.20 & 20 & A & Dark topsoil with many roots & 20.87 & 0.0397 & 0.0101 \\
\hline SCT3 1.30 & 30 & $\mathrm{AB}$ & Dark subsoil w/ nodules from above and roots & 22.22 & 0.0443 & 0.0145 \\
\hline SCT3 1.55 & 55 & $\mathrm{AB}$ & Dark subsoil w/ nodules from below and roots & 27.87 & 0.0421 & - \\
\hline SCT3 1.70 & 70 & $\mathrm{~B}$ & Mottled subsoil with feldsbar, clay films and roots & 26.37 & 0.0349 & - \\
\hline SCT3 1.90 & 90 & $\mathrm{~B}$ & Dark subsoil with few pebbles and roots & 0.68 & 0.0203 & 0.0128 \\
\hline SCT3 1.100 & 100 & $\mathrm{~B}$ & Highly mottled subsoil & 1.03 & 0.0340 & 0.0052 \\
\hline SCT4 1.10 & 10 & A & Dark topsoil with roots & 40.69 & 0.3205 & 0.0191 \\
\hline SCT4 1.20 & 20 & $\mathrm{AB}$ & Dark topsoil with roots & 31.51 & 0.3995 & - \\
\hline SCT4 1.28 & 28 & $\mathrm{AB}$ & Dark topsoil with roots & 33.39 & 0.3873 & 0.1095 \\
\hline SCT4 1.40 & 40 & $\mathrm{~B}$ & Dark subsoil with clay films and roots & 31.20 & 0.2277 & 0.1286 \\
\hline SCT4 1.60 & 60 & $\mathrm{~B}$ & Dark subsoil with clay films and roots & 7.86 & 0.1725 & 0.0034 \\
\hline SCT4 1.80 & 80 & $\mathrm{~B}$ & Dark subsoil with mottling and roots on ped faces & 0.00 & 0.1120 & - \\
\hline SCT4 1.100 & 100 & $\mathrm{~B}$ & Dark subsoil with mottling & 0.00 & 0.1135 & - \\
\hline
\end{tabular}


table 5.xls

\begin{tabular}{|c|c|c|c|c|c|c|c|}
\hline Profile ID & $\begin{array}{c}\text { Basal } \\
\text { Depth } \\
(\mathrm{cm})\end{array}$ & $\begin{array}{c}\text { Horizon } \\
\text { ID }\end{array}$ & Sample Description & $\begin{array}{c}>2-\mathbf{m m} \\
\text { Fraction } \\
\quad(\%)\end{array}$ & $\begin{array}{c}<2-\mathrm{mm} \\
\text { Average } \\
\text { Fraction } \\
\quad(\%)\end{array}$ & $\begin{array}{c}<2-\mathrm{mm} \\
\mathrm{SD}(\mathrm{n}=2) \\
(\%)\end{array}$ & \\
\hline SCT5 1.10 & 10 & A & Dark topsoil with roots & 20.93 & 0.0629 & 0.0010 & \\
\hline SCT5 1.20 & 20 & A & Dark topsoil with roots & 19.21 & 0.0419 & - & \\
\hline SCT5 1.37 & 37 & $\mathrm{AB}$ & Dark subsoil with roots & 20.68 & 0.0975 & 0.0133 & \\
\hline SCT5 1.55 & 55 & B & Dark subsoil w/red pebbles and v.fine roots along ped faces & 13.08 & 0.0322 & 0.0025 & \\
\hline SCT5 1.80 & 80 & B & Dark subsoil with clay films, common red pebbles & 3.23 & 0.0203 & - & \\
\hline \multirow[t]{2}{*}{ SCT5 1.100} & 100 & $\mathrm{~B}$ & Dark subsoil with common v.fine roots along ped faces & 0.00 & 0.0293 & - & \\
\hline & & & & & & $\begin{array}{c}\text { Average } \\
\text { SD }\end{array}$ & 0.0291 \\
\hline
\end{tabular}




\begin{tabular}{|c|c|c|c|c|c|c|c|c|c|c|c|c|}
\hline Terrace & $\begin{array}{c}\text { Sample } \\
\text { ID }\end{array}$ & $\begin{array}{l}\text { Basal } \\
\text { Depth } \\
\text { (pm) }\end{array}$ & $\begin{array}{c}\text { Description } \\
\text { Interval (cm) }\end{array}$ & Site Location & Sample Description & Roots Present & $\begin{array}{l}\text { Dry Munsell } \\
\text { Color }\end{array}$ & $\begin{array}{l}\text { Dry Munsell } \\
\text { Color }\end{array}$ & $\begin{array}{l}\text { Dry Munsell } \\
\text { Color }\end{array}$ & Texture & Stickiness & Plasticity \\
\hline \multicolumn{2}{|c|}{ Wilder beach } & 10 & $0-10$ & & Colorful beach sand, $\mathrm{pH}=7.2$ & none & $10 \mathrm{YR} 7 / 2$ & & & sand & not sticky & not plastic \\
\hline SCT1 & $1 \mathrm{a} .20$ & 20 & $0-6$ & By corner of Pelton and Eucalytus, go into park through & Brown topsoil with sm piece of concrete, roots & very fine, fine, medium & $10 \mathrm{YR} 6 / 2$ & & & loam & slightly sticky & slightly plastic \\
\hline SCT1 & $1 \mathrm{a} .35$ & 35 & $20-27$ & Monach resting area, go left, go between trees look for & Brown topsoil, roots & fine & $10 \mathrm{YR} 5 / 3$ & & & loam & slightly sticky & slightly plastic \\
\hline SCT1 & $1 \mathrm{a} .58$ & 58 & $47-58$ & sight on right, may be a path. Auger description of gas & Brown subsoil with few small pebbles & none & $10 \mathrm{YR} 4 / 4$ & & & loam & 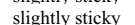 & 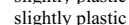 \\
\hline SCT1 & 1a.67 & 67 & $58-67$ & sampler hole. & $\begin{array}{l}\text { Light brown subsoil } \\
\text {. }\end{array}$ & none & $10 \mathrm{YR} 4 / 4$ & 10 YR5 $5 / 6$ & & silt loam & slightly sticky & slightly plastic \\
\hline SCT1 & $1 \mathrm{a} .90$ & 90 & $67-78$ & & Light brown subsoil & none & $10 \mathrm{YR} 6 / 3$ & $10 \mathrm{YR} 5 / 8$ & 10YR5/6 & loam & slightly sticky & slightly plastic \\
\hline SCT1 & 1a.112 & 112 & $90-100$ & & Grey brown subsoil & none & $10 \mathrm{YR} 7 / 3$ & $10 \mathrm{YR} 6 / 8$ & & sandy loam & not sticky & not plastic \\
\hline SCT1 & 1a. 121 & 121 & $112-121$ & & Grey brown subsoil with maganese stains & none & $10 \mathrm{YR} 7 / 3$ & 10YR6/8 & & loam & slightly sticky & slightly plastic \\
\hline SCT1 & 1a. 160 & 160 & $121-131$ & & Light brown subsoil & none & 10YR6/4 & & & loam & slightly sticky & slightly plastic \\
\hline SCT1 & 1a.187 & 187 & 165-171 & & Coarse sand, many colors of pebbles, $\mathrm{C}$ horizon & none & 10YR $4 / 4$ & & & loamy sand & not sticky & not plastic \\
\hline SCT1 & 1a.197 & 197 & 187-197 & & Fine sand, $\mathrm{C}$ horizon & none & 10YR $4 / 4$ & & & sand & not sticky & not plastic \\
\hline SCT1 & 2a.10 & 10 & $0-6$ & By corner of Pelton and Eucalytus, go into park through & Brown topsoil with roots & very fine, fine, medium & $10 \mathrm{YR} 5 / 2$ & & & silt loam & slightly sticky & slightly plastic \\
\hline SCT1 & 2a.19 & 19 & 19-Oct & Monach resting area, go right, on left will be the site. & & fine & $10 \mathrm{YR} 3 / 3$ & & & silt loam & slightly sticky & slightly plastic \\
\hline SCT1 & $2 \mathrm{a} .45$ & 45 & $19-30$ & Auger description of $8 \mathrm{ft}$ water sampler hole. & Dark brown subsoil with small pebbles, roots & fine & $10 \mathrm{YR} 4 / 2$ & & & loam & slightly sticky & 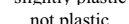 \\
\hline SCT1 & $2 \mathrm{a} .50$ & 50 & $40-50$ & & Brown subsoil with roots & fine & $10 \mathrm{YR} 5 / 3$ & & & sandy loam & not sticky & not plastic \\
\hline SCT1 & 2a.74 & 74 & $50-60$ & & Brown subsoil & fine & $10 \mathrm{YR} 5 / 3$ & & & clay & very sticky & very plastic \\
\hline SCT1 & 2a.92 & 92 & $74-84$ & & Dark brown subsoil & none & $10 \mathrm{YR} 4 / 3$ & & & clay & very sticky & verv plastic \\
\hline SCT1 & 2a. 104 & 104 & $92-96$ & & Yellow, brown subsoil with sm lenses of red, brown & none & $10 \mathrm{YR} 5 / 6$ & $7.5 \mathrm{YR} 3 / 4$ & & clay loam & sticky & $\begin{array}{l}\text { plastic } \\
\text { plic }\end{array}$ \\
\hline SCT1 & 2a.118 & 118 & 104-109 & & Very gritty, brown subsoil & none & $10 \mathrm{YR} 5 / 6$ & & & sandy loam & not sticky & slightly plastic \\
\hline SCT1 & 2a. 142 & 142 & $118-123$ & & Brown subsoil with maganese & none & $10 \mathrm{YR} 5 / 6$ & & & sandy loam & not sticky & not plastic \\
\hline SCT1 & 2a.172 & 172 & 142-152 & & Brown subsoil & none & $10 \mathrm{YR} 5 / 4$ & & & sandy loam & not sticky & not plastic \\
\hline SCT1 & 2a. 183 & 183 & 172-183 & & Brown subsoil & none & $10 \mathrm{YR} 4 / 4$ & & & sandy loam & not sticky & not plastic \\
\hline SCT1 & 2a.195 & 195 & 183-195 & & Brown C horizon & none & 10YR4/4 & & & loamy sand & not sticky & not plastic \\
\hline SCT1 & 2a.202 & 202 & $195-202$ & & Dark brown C horizon & none & $10 \mathrm{YR} 2 / 3$ wet & & & loamy sand & not sticky & not plastic \\
\hline SCT2 & 1a.23 & 23 & $0-11$ & South of sample enclosure in Wilder Ranch, augar site & Brown topsoil with roots & fine, medium & $10 \mathrm{YR} 4 / 3$ & & & silt loam & not sticky & slightly plastic \\
\hline SCT2 & 1a.45 & 45 & $23-36$ & 1 , annual grass & Brown topsoil with roots & fine & $10 \mathrm{YR} 4 / 6$ & & & silt loam & slightly sticky & slightly plastic \\
\hline SCT2 & 1. .54 & 54 & $45-54$ & & Brown subsoil with small pebbles & none & 10YR5/6 & & & silt loam & slightly sticky & plastic \\
\hline SCT2 & 1a. 84 & 84 & $54-63$ & & Brown, orange subsoil & none & 10YR6/8 & $10 \mathrm{YR} 4 / 4$ & & silt clay loam & sticky & plastic \\
\hline SCT2 & 1a.112 & 112 & $84-94$ & & Grey, orange subsoil with feldspar and pebbles & none & 10YR4/1 & $10 \mathrm{YR} 6 / 8$ & & silt clay loam & sticky & very plastic \\
\hline SCT2 & 1a.139 & 139 & $112-123$ & & Grey, orange subsoil with feldspar & none & $10 \mathrm{YR} 6 / 8$ & 10YR $4 / 1$ & & silt clay loam & sticky & plastic \\
\hline SCT2 & 1a.152 & 152 & 139-145 & & Orange, gritty subsoil & none & 10YR5/8 & & & clay loam & sticky & plastic \\
\hline SCT2 & 1a.171 & 171 & $152-160$ & & Orange subsoil & none & 7.5YR $6 / 6$ & & & clay loam & sticky & plastic \\
\hline $\mathrm{SCT} 2$ & 1a.192 & 192 & 171-180 & & Orange, grey subsoil & none & 10YR $7 / 8$ & & & clay loam & sticky & plastic \\
\hline SCT2 & 1a.199 & 199 & 192-199 & & Orange, grey subsoil & none & $10 \mathrm{YR} 7 / 8$ & & & loam & slightly sticky & slightly plastic \\
\hline SCT2 & 2a. 13 & 13 & $0-13$ & North of sample enclosure $\sim 2 \mathrm{~m}$, on east side & & & $10 \mathrm{YR} 5 / 3$ & & & loam & slightly sticky & \\
\hline SCT2 & $2 \mathrm{a} .34$ & 34 & $13-24$ & & Brown topsoil with roots & very fine, fine, medium & $10 \mathrm{YR} 4 / 4$ & & & loam & slightly sticky & slightly plastic \\
\hline SCT2 & $2 \mathrm{a} .51$ & 51 & $34-43$ & & Dark brown topsoil with few small pebbles, roots & fine & 10 YR5 $5 / 4$ & 10 YR3 34 & & loam & slightly sticky & 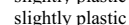 \\
\hline SCT2 & 2a.66 & 66 & $51-58$ & & Brown subsoil with roots & very fine & $10 \mathrm{YR} 5 / 6$ & & & clay loam & slightly sticky & plastic \\
\hline SCT2 & $2 a .86$ & 86 & $66-76$ & & Brown, orange subsoil & none & $10 \mathrm{YR} 5 / 6$ & $7.5 \mathrm{YR} 5 / 8$ & & clay loam & sticky & plastic \\
\hline SCT2 & 2a. 103 & 103 & $86-94$ & & Orange, brown, grey subsoil with small pebbles & none & $10 \mathrm{YR} 4 / 3$ & 7.5 YR $5 / 8$ & & clay & very sticky & very plastic \\
\hline SCT2 & 2a. 115 & 115 & 103-111 & & Orange, grey subsoil with lots of chalky feldspar & none & 10YR $5 / 1$ & $7.5 \mathrm{YR} 5 / 8$ & & clay & very sticky & very plastic \\
\hline SCT2 & 2a. 145 & 145 & $115-127$ & & Grey, orange subsoil with lots of chalky feldspar & none & $10 \mathrm{YR} 5 / 8$ & $7.5 \mathrm{YR} 7 / 2$ & $2.5 \mathrm{YR} 4 / 1$ & clay & very sticky & very plastic \\
\hline SCT2 & 2a.181 & 181 & 145-155 & & Grey, orange, lt.brown subsoil with lots of chalky feldpar & none & $10 \mathrm{YR} 5 / 8,5 / 4$ & 7.5 YR7/2 & $2.5 \mathrm{YR} 4 / 1$ & clay & very sticky & very plastic \\
\hline SCT2 & 2a.191 & 191 & 181-189 & & Light brown, orange subsoil & none & 10YR 5/6 & $7.5 \mathrm{YR} 7 / 2$ & & clay loam & sticky & plastic \\
\hline SCT2 & 2a.200 & 200 & $191-200$ & & Light brown subsoil & none & 10YR5/6 & & & clay loam & sticky & plastic \\
\hline SCT3 & $1 \mathrm{a} .26$ & 26 & $0-9$ & North $\sim 3 \mathrm{~m}$ of sample enclosure & Light brown topsoil with roots, a few small pebbles & very fine, fine, medium & $10 \mathrm{YR} 5 / 3$ & & & silt loam & slightly sticky & slightly plastic \\
\hline SCT3 & 1a.45 & 45 & $26-37$ & & Yellow brown subsoil w/medium ( $2 \mathrm{cmwide}$ ) nodules, & very fine, fine, medium & $10 \mathrm{YR} 5 / 6$ & & & silt loam & $\begin{array}{l}\text { slightly sticky } \\
\text { sits }\end{array}$ & slightly plastic \\
\hline SCT3 & $1 \mathrm{a} .56$ & 56 & $45-48$ & & Orange, brown subsoil with few small pebbles, roots & very fine & $10 \mathrm{YR} 5 / 8$ & & & silt clay loam & slightly sticky & \\
\hline SCT3 & 1a.70 & 70 & $56-60$ & & Brown, orange, grey subsoil & none & $10 \mathrm{YR} 5 / 4$ & 10YR $5 / 1$ & & clay loam & & plastic \\
\hline SCT3 & 10.84 & 84 & $70-80$ & & $\begin{array}{l}\text { Brown, orange, grey, red subsoil } \\
\text { Bef }\end{array}$ & none & $10 \mathrm{YR} 4 / 45 / 1$ & $7.5 \mathrm{YR} 5 / 8$ & $5 \mathrm{YR} 4 / 8$ & clay loam & sticky & plastic \\
\hline SCT3 & 1a.110 & 110 & $84-91$ & & Orange, grey subsoil with lots of chalky spots & none & $10 \mathrm{YR} 4 / 1$ & $7.5 \mathrm{YR} 5 / 8$ & & clay & sticky & very plastic \\
\hline SCT3 & 1a. 142 & 145 & $110-117$ & & Orange, grey, red subsoil with lots of chalky spots & none & $7.5 \mathrm{YR} 5 / 1$ & $7.5 \mathrm{YR} 5 / 8$ & $5 Y R 4 / 1$ & clay & sticky & very plastic \\
\hline SCT3 & 1a. 150 & 150 & $142-147$ & & Orange, grey subsoil with white chalky spots & none & 7.5 YR $5 / 8$ & $2.5 \mathrm{Y} 4 / 1$ & Blue grey & clay loam & sticky & plastic \\
\hline SCT3 & 1a.160 & 160 & 150-152 & & Orange, brown, grey subsoil with white chalky spots & none & $10 \mathrm{YR} 5 / 4$ & 10YR6/1 & $7.5 \mathrm{YR} 5 / 8$ & clay loam & sticky & very plastic \\
\hline SCT3 & 1a.184 & 184 & 160-164 & & Orange, brown, grey subsoil & none & 10YR $4 / 6$ & 10YR6/1 & $7.5 \mathrm{YR} 5 / 8$ & clay loam & sticky & plastic \\
\hline SCT3 & $1 \mathrm{a} .200$ & 200 & $184-190$ & & Orange, brown subsoil & none & $7.5 \mathrm{YR} 4 / 6$ & & & clay loam & sticky & plastic \\
\hline
\end{tabular}




\begin{tabular}{|c|c|c|c|c|c|c|c|c|c|c|c|c|}
\hline Terrace & $\begin{array}{c}\text { Sample } \\
\text { ID }\end{array}$ & $\begin{array}{l}\text { Basal } \\
\text { Depth } \\
\text { (cm) }\end{array}$ & $\begin{array}{c}\text { Description } \\
\text { Interval (cm) }\end{array}$ & Site Location & Sample Description & Roots Present & $\begin{array}{l}\text { Dry Munsell } \\
\text { Color }\end{array}$ & $\begin{array}{l}\text { Dry Munsell } \\
\text { Color }\end{array}$ & $\begin{array}{l}\text { Dry Munsell } \\
\text { Color }\end{array}$ & Texture & Stickiness & Plasticity \\
\hline SCT3 & $2 \mathrm{a} .32$ & 32 & 21-Oct & \multirow[t]{11}{*}{ East $\sim 5 \mathrm{~m}$ of sample enclosure } & Brown topsoil with roots & very fine, fine & $10 \mathrm{YR} 4 / 4$ & & & silt loam & slightly sticky & slightly plastic \\
\hline SCT3 & $2 \mathrm{a} .40$ & 40 & $32-40$ & & Brown topsoil with lots of pebbles, roots & very fine, fine & $10 \mathrm{YR} 4 / 4$ & & & silt loam & slightly sticky & slightly plastic \\
\hline SCT3 & $2 a .57$ & 57 & $40-50$ & & $\begin{array}{l}\text { Yellow, brown subsoil with roots, } \\
\text { Yools }\end{array}$ & very fine & 10YR5/6 & & & silt loam & $\begin{array}{l}\text { slightly sticky } \\
\text { slist }\end{array}$ & $\begin{array}{l}\text { slightly plastic } \\
\text { slats }\end{array}$ \\
\hline SCT3 & $2 \mathrm{a} .78$ & 78 & $57-64$ & & Brown subsoil, large noduel at $78 \mathrm{~cm}$, roots & fine & $10 \mathrm{YR} 5 / 4$ & 10YR6/8 & & silt clay loam & $\begin{array}{l}\text { sticky } \\
\text { stick } y\end{array}$ & $\begin{array}{l}\text { plastic } \\
\text { plic }\end{array}$ \\
\hline SCT3 & $2 \mathrm{a} .90$ & 90 & $78-87$ & & Dark brown, orange subsoil with small pebbles & none & $10 \mathrm{YR} 4 / 4$ & $10 \mathrm{YR} 3 / 2$ & $7.5 \mathrm{YR} 5 / 8$ & clay & sticky & plastic \\
\hline SCT3 & 2a.107 & 107 & $90-97$ & & Orange, red, grey subsoil with small pebbles & none & $10 \mathrm{YR} 5 / 1$ & $10 \mathrm{YR} 5 / 8$ & $5 \mathrm{YR} 3 / 6$ & clay & very sticky & very plastic \\
\hline SCT3 & 2a. 117 & 117 & $107-111$ & & & none & $2.5 \mathrm{YR} 4 / 8$ & $2.5 \mathrm{YR} 4 / 1$ & 7.5 YR $5 / 8$ & clay & very sticky & very plastic \\
\hline SCT3 & 2a.140 & & $117-126$ & & Brown, orange subsoil with lots of small pebbles & none & $10 \mathrm{YR} 5 / 1$ & 7.5 YR $5 / 8$ & & clay & very sticky & very plastic \\
\hline SCT3 & 2a. 160 & 150 & $140-150$ & & Brown, red subsoil & none & 10YR5/1 & $2.5 \mathrm{YR} 3 / 6$ & & clay & very sticky & very plastic \\
\hline SCT3 & 2a. 190 & 190 & $160-168$ & & Red subsoil with small pebbles & none & $5 Y \mathrm{YR} 4 / 6$ & & & clay loam & sticky & plastic \\
\hline SCT3 & 2a.203 & 203 & $190-193$ & & Brown, orange subsoil with small pebbles and nodules & none & $10 \mathrm{YR} 5 / 4$ & $7.5 \mathrm{YR} 4 / 6$ & & clay loam & 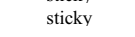 & plastic \\
\hline SCT3 & $3 a .17$ & 17 & $0-10$ & \multirow[t]{10}{*}{$5 \mathrm{~m}$ north of sample enclosure. Annual grass and oaks } & Brown topsoil with roots and small pebbles & very fine, fine & $10 \mathrm{YR} 4 / 4$ & & & silt loam & slightly sticky & slightly plastic \\
\hline SCT3 & $3 \mathrm{a} .28$ & 28 & $17-28$ & & Brown topsoil with roots & very fine & $10 \mathrm{YR} 5 / 8$ & & & silt loam & slightly sticky & $\begin{array}{l}\text { slightly plastic } \\
\text { s. }\end{array}$ \\
\hline SCT3 & $3 \mathrm{a} .40$ & 40 & $28-36$ & & Brown subsoil with small pebbles, nodules, roots & very fine & $10 \mathrm{YR} 5 / 6$ & & & silt loam & slightly sticky & slightly plastic \\
\hline SCT3 & $3 a .63$ & 63 & $40-53$ & & $\begin{array}{l}\text { Brown, orange subsoil with roots } \\
\text { Beules, }\end{array}$ & very fine & $10 \mathrm{YR} 5 / 4$ & $7.5 \mathrm{YR} 5 / 8$ & & silt clay loam & 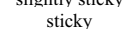 & $\begin{array}{l}\text { plastic } \\
\text { plic }\end{array}$ \\
\hline SCT3 & 3a.72 & 72 & 63-68 & & $\begin{array}{l}\text { Brown, orange subsoil with small pebbles, nodules, roots } \\
\text { Babs }\end{array}$ & very fine & $10 \mathrm{YR} 6 / 4$ & 7.5YR5/8 & & clay & 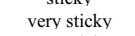 & very plastic \\
\hline SCT3 & $3 a .84$ & 84 & $72-80$ & & $\begin{array}{l}\text { Brown, orange subsoil w/white chalky feldspar and sm.pebbles }\end{array}$ & none & $10 \mathrm{YR} 5 / 1$ & $7.5 \mathrm{YR} 5 / 8$ & & clay & very sticky & very plastic \\
\hline SCT3 & $3 \mathrm{a} .95$ & 95 & $84-91$ & & Grey, orange subsoil & none & 10YR $4 / 1$ & $7.5 \mathrm{YR} 5 / 8$ & & clay & very sticky & very plastic \\
\hline SCT3 & $3 \mathrm{a} .110$ & 110 & $95-100$ & & Grey, brown, orange subsoil & none & $10 \mathrm{YR} 4 / 1$ & $10 \mathrm{YR} 6 / 85 / 4$ & $7.5 \mathrm{YR} 5 / 8$ & clay & very sticky & very plastic \\
\hline SCT3 & 3a. 128 & 128 & $110-117$ & & Brown, red, orange subsoil, at $112 \mathrm{~cm}$ white chaky felspar & none & 10YR5/1 & 5YR4/8 & 7.5YR $5 / 8$ & silt clay loam & sticky & plastic \\
\hline SCT3 & 3.150 & 150 & $128-150$ & & Brown, red, orange subsoil & none & 10YR6/1 & $5 \mathrm{YR} 4 / 8$ & $7.5 \mathrm{YR} 5 / 8$ & silt clay loam & sticky & plastic \\
\hline SCT4 & $1 \mathrm{a} .24$ & 24 & $0-12$ & \multirow{9}{*}{$\begin{array}{l}\text { South of sample enclosure in Wilder Ranch, augar site } \\
1 \text {, brush, little annual grass }\end{array}$} & Light brown topsoil with roots & fine, medium & $10 \mathrm{YR} 4 / 4$ & & & loam & not sticky & slightly plastic \\
\hline SCT4 & 1a.45 & 45 & $24-36$ & & Light brown topsoil with roots & fine & 7.5YR4/6 & & & loam & not sticky & slightly plastic \\
\hline SCT4 & 1a.54 & 54 & $45-54$ & & Orange, brown subsoil with sm pebbles & none & $10 \mathrm{YR} 4 / 6$ & & & loam & not sticky & slightly plastic \\
\hline SCT4 & 1a.74 & 74 & $54-64$ & & Orange, red subsoil & none & $2.5 \mathrm{YR} 3 / 4$ & $7.5 \mathrm{YR} 4 / 6$ & & silt loam & sticky & plastic \\
\hline SCT4 & 1a. 110 & 110 & $74-83$ & & Orange, red, grey subsoil & none & $2.5 \mathrm{YR} 3 / 4$ & $2.5 \mathrm{YR} 5 / 2$ & $7.5 \mathrm{YR} 4 / 6$ & silt loam & sticky & plastic \\
\hline SCT4 & 1a. 135 & 135 & $110-118$ & & Reddish, orange, grey subsoil & none & 10YR6/1 & $2.5 \mathrm{YR} 3 / 6$ & $5 \mathrm{YR} 4 / 6$ & silt clay loam & sticky & very plastic \\
\hline SCT4 & 1..157 & 157 & $135-147$ & & Grey, red, orange subsoil few sm pebbles & none & 10YR6/1 & $2.5 \mathrm{YR} 3 / 6$ & $5 \mathrm{YR} 4 / 6$ & silty clay & sticky & very plastic \\
\hline SCT4 & 1a.171 & 171 & $\begin{array}{l}157-166 \\
171182\end{array}$ & & Orange, grey, red subsoil & none & 7.5YR $5 / 8$ & $10 \mathrm{YR} 7 / 1$ & $2.5 \mathrm{YR} 3 / 6$ & silt clay loam & sticky & plastic \\
\hline $\begin{array}{l}\text { SCT4 } \\
\text { SCT4 }\end{array}$ & $\begin{array}{l}\text { 1a. } 195 \\
\text { 1a.204 }\end{array}$ & $\begin{array}{l}195 \\
204\end{array}$ & $\begin{array}{l}171-182 \\
195-204\end{array}$ & & $\begin{array}{l}\text { Orange, grey, red subsoil } \\
\text { Orange, grey subsoil with chalky feldspar }\end{array}$ & $\begin{array}{c}\text { none } \\
\text { none }\end{array}$ & $\begin{array}{l}7.5 \mathrm{YR} 5 / 8 \\
10 \mathrm{Y} 6 / 6\end{array}$ & 10YR $7 / 1$ & $2.5 \mathrm{YR} 3 / 6$ & silt clay loam & sticky & $\begin{array}{c}\text { plastic } \\
\text { nlastic }\end{array}$ \\
\hline & & & (1) & \multirow{9}{*}{$\begin{array}{l}\text { East of sample enclosure in Wilder Ranch, augar site 2, } \\
\text { brush, little annual grass }\end{array}$} & & Honic & $101 \pi 0 \%$ & 10YR $7 / 1$ & & nd & stucky & plasitc \\
\hline SCT4 & 2a.29 & 29 & $0-13$ & & Light brown topsoil with roots & fine, medium & $10 \mathrm{YR} 4 / 4$ & & & loam & not sticky & not plastic \\
\hline SCT4 & $2 \mathrm{a} .46$ & 46 & $29-39$ & & Brown topsoil with roots & very fine & 10YR4/6 & & & loam & not sticky & slightly plastic \\
\hline SCT4 & $\begin{array}{l}2 \mathrm{a} .64 \\
2.81\end{array}$ & 64 & $\begin{array}{l}46-56 \\
64.74\end{array}$ & & Orange, brown, red subsoil with sm pebbles & very fine & $10 \mathrm{YR} 5 / 6$ & & & silt loam & slightly sticky & $\begin{array}{l}\text { slightly plastic } \\
\text { slats }\end{array}$ \\
\hline SCT4 & $2 \mathrm{a} .81$ & 81 & $64-74$ & & Brown subsoil with feldspar pebbles & none & 10YR $4 / 6$ & $10 \mathrm{YR} 5 / 6$ & $5 \mathrm{YR} 5 / 8$ & silt loam & slightly sticky & $\begin{array}{l}\text { slightly plastic } \\
\text { s. }\end{array}$ \\
\hline SCT4 & 2a.106 & 106 & $81-90$ & & Orange, grey subsoil with lots of $\mathrm{sm}$. grained pebbles & none & 10YR5/1 & 7.5YR5/8 & & silt clay loam & sticky & plastic \\
\hline SCT4 & 2a.125 & 125 & $106-114$ & & Red, orange, grey subsoil with lots of pebbles & none & $2.5 \mathrm{YR} 3 / 6$ & 10YR7/1 & & silt loam & slightly sticky & slightly plastic \\
\hline SCT4 & 2a.135 & 135 & $125-135$ & & Gritty red C horizon with small area of yellow & none & $5 \mathrm{YR} 4 / 8$ & $2.5 \mathrm{Y} 8 / 6$ & & sandy loam & not sticky & not plastic \\
\hline SCT4 & 2a.137 & 137 & & & Hit a rock & & & & & & & \\
\hline SCT4 & 3a.17 & 17 & $0-10$ & \multirow{11}{*}{$\begin{array}{l}\text { North of sample enclosure. Trees (oaks), brush, small } \\
\text { annual grass }\end{array}$} & Light brown topsoil with roots & very fine, fine & $10 \mathrm{YR} 4 / 4$ & & & loam & slightly sticky & not plastic \\
\hline SCT4 & $3 \mathrm{a} .30$ & 30 & $17-26$ & & Brown subsoil with small pebbles, wood piece, roots & medium & 10YR5/6 & & & loam & slightly sticky & slightly plastic \\
\hline SCT4 & $3 \mathrm{a} .40$ & 40 & $30-40$ & & $\begin{array}{l}\text { Brown subsoil with small amounts of Fe stains, roots }\end{array}$ & fine & $10 \mathrm{YR} 4 / 6$ & & & loam & slightly sticky & $\begin{array}{l}\text { slightly plastic } \\
\text { s. }\end{array}$ \\
\hline SCT4 & $3 \mathrm{a} .63$ & 63 & $40-48$ & & $\begin{array}{l}\text { Brown, orange subsoil with roots, small nodules } \\
\text { Dodula }\end{array}$ & very fine & $10 \mathrm{YR} 4 / 6$ & & & clay loam & 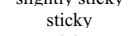 & 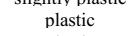 \\
\hline SCT4 & $3 a .88$ & 88 & $63-74$ & & Grey, orange, red subsoil with lots of small pebbles & none & $10 \mathrm{YR} 4 / 2$ & $10 \mathrm{YR} 5 / 8$ & $5 Y \mathrm{YR} 4 / 8$ & clay loam & sticky & plastic \\
\hline SCT4 & $3 a .100$ & 100 & $88-95$ & & Orange, grey, red subsoil with thin clay films & none & $10 \mathrm{YR} 5 / 1$ & $5 \mathrm{YR} 5 / 8$ & $7 \mathrm{YR} 5 / 8$ & clay & very sticky & very plastic \\
\hline SCT4 & 3a.109 & 109 & $100-109$ & & Orange, red, grey subsoil & none & 10YR6/1 & $2.5 \mathrm{YR} 5 / 8$ & $7 Y R 5 / 8$ & clay & very sticky & very plastic \\
\hline SCT4 & $3 a .140$ & 140 & $109-114$ & & Red, orange, grey subsoil with small pebbles & none & 10YR6/1 & $5 Y R 4 / 8$ & 10YR6/8 & clay & very sticky & very plastic \\
\hline SCT4 & 3a.170 & 170 & $140-147$ & & Red, grey, yellow, orange subsoil with coarse roots at $157 \mathrm{~cm}$ & none & 10YR6/1 & $5 \mathrm{YR} 4 / 8$ & $2.5 \mathrm{YR} 7 / 8$ & clay & very sticky & very plastic \\
\hline SCT4 & 3a.198 & 198 & $170-175$ & & Orange, grey, subsoil with a small rock & none & 10YR $7 / 1$ & 10YR6/8 & & clay loam & sticky & plastic \\
\hline SCT4 & $3 \mathrm{a} .206$ & 206 & $198-206$ & & Orange, grey subsoil with small rocks & none & 10YR7/1 & 10YR6/8 & & clay loam & sticky & plastic \\
\hline SCT5 & 10.24 & 24 & $0-12$ & \multirow{10}{*}{$\begin{array}{l}\text { Site off Backranch Rd, Imeter southwest of sampler } \\
\text { W30 }\end{array}$} & Dark brown topsoil with roots & very fine, fine & 10YR3/3 & & & silt lo & not sticky & \\
\hline SCT5 & $1 \mathrm{a} .45$ & 45 & 24-37 & & & & $10 \mathrm{YR} 4 / 4$ & & & silt loam & not sticky & slightly plas \\
\hline SCT5 & 1a.60 & 60 & $45-54$ & & Light brown subsoil with orange, red are & fine & 10 YR5 $5 / 6$ & $10 \mathrm{YR} 4 / 3$ & $2.5 \mathrm{YR} 4 / 8$ & silt clay loam & sticky & plastic \\
\hline SCT5 & 10.87 & 87 & $60-66$ & & Grey, orange, red subsoil with thin clay films, roots & very fine & $10 \mathrm{YR} 4 / 2$ & $2.5 \mathrm{YR} 4 / 8$ & $7.5 \mathrm{YR} 6 / 8$ & silt clay loam & sticky & very plastic \\
\hline SCT5 & 1a.114 & 114 & $87-96$ & & Grey, orange, red subsoil with thin clay films, roots & very fine & $10 \mathrm{YR} 5 / 1$ & $7.5 \mathrm{YR} 5 / 8$ & $2.5 \mathrm{YR} 4 / 8$ & silt clay loam & sticky & very plastic \\
\hline SCT5 & 1a. 133 & 133 & $114-120$ & & Grey, orange, red subsoil with roots & very fine & $2.5 \mathrm{YR} 3 / 6$ & $7.5 \mathrm{YR} 5 / 8$ & 10YR $5 / 1,6 / 1$ & clay loam & sticky & very plastic \\
\hline SCT5 & 1a. 149 & 149 & $133-140$ & & Grey, orange subsoil & none & 10YR5/1 & 10YR6/1 & 7.5 YR $5 / 8$ & clay loam & sticky & very plastic \\
\hline SCT5 & 1a.177 & 177 & $149-152$ & & Loose orange, grey subsoil & none & 10YR $7 / 1$ & $10 \mathrm{YR} 6 / 8$ & & loam & slightly sticky & plastic \\
\hline SCT5 & 1a.193 & 193 & $177-182$ & & Orange, grey with some cemented grey areas, $\mathrm{C}$ horizon & none & 10YR6/8 & $10 \mathrm{YR} 7 / 1$ & & sandy loam & not sticky & not plastic \\
\hline SCT5 & $1 \mathrm{a} .200$ & 200 & $193-200$ & & Orange, grey C horizon & none & $10 \mathrm{YR} 6 / 8$ & 10YR $7 / 1$ & & sandy loam & not sticky & not plastic \\
\hline
\end{tabular}




\begin{tabular}{|c|c|c|c|c|c|c|c|c|c|c|c|c|}
\hline Terrace & $\begin{array}{c}\text { Sample } \\
\text { ID }\end{array}$ & $\begin{array}{l}\text { Basal } \\
\text { Depth } \\
\text { (cm) }\end{array}$ & $\begin{array}{c}\text { Description } \\
\text { Interval (cm) }\end{array}$ & Site Location & Sample Description & Roots Present & $\begin{array}{l}\text { Dry Munsell } \\
\text { Color }\end{array}$ & $\begin{array}{l}\text { Dry Munsell } \\
\text { Color }\end{array}$ & $\begin{array}{c}\text { Dry Munsell } \\
\text { Color }\end{array}$ & Texture & Stickiness & Plasticity \\
\hline SCT5 & 2a.16 & 16 & $0-10$ & Site off Backranch $\mathrm{Rd}, \sim 5$ meter north of drip rain guage & Brown topsoil with few nodules, roots & very fine, medium & $10 \mathrm{YR} 4 / 3$ & & & loam & slightly sticky & plastic \\
\hline SCT5 & $2 \mathrm{a} .34$ & 34 & $16-23$ & & Brown topsoil with few nodules, roots & fine & $10 \mathrm{YR} 4 / 4$ & & & loam & slightly sticky & slightly plastic \\
\hline SCT5 & $2 \mathrm{a} .55$ & 55 & 34-42 & & Brown, red subsoil with roots & very fine & $10 \mathrm{YR} 4 / 4$ & $10 \mathrm{YR} 4 / 8$ & & silt clay loam & slightly sticky & slightly plastic \\
\hline SCT5 & $2 \mathrm{a} .66$ & 66 & $51-61$ & & Brown, orange subsoil with roots & very fine & $10 \mathrm{YR} 3 / 2$ & $10 \mathrm{YR} 5 / 8$ & & silt clay loam & slightly sticky & slightly plastic \\
\hline SCT5 & $2 \mathrm{a} .85$ & 85 & $66-71$ & & Brown, red, orange subsoil with thin clay films, roots & very fine & $10 \mathrm{YR} 3 / 2$ & $7.5 \mathrm{YR} 5 / 8$ & $5 \mathrm{YR} 3 / 2$ & silt clay loam & slightly sticky & slightly plastic \\
\hline SCT5 & 2a. 111 & 111 & $85-94$ & & Grey, orange, red subsoil with thin clay films, roots & very fine & 10YR $5 / 1$ & $7.5 \mathrm{YR} 5 / 8$ & $5 \mathrm{YR} 3 / 2$ & silty clay & sticky & very plastic \\
\hline SCT5 & $2 \mathrm{a} .135$ & 135 & $111-121$ & & Grey, orange, red subsoil & none & 10YR $5 / 1$ & $7.5 \mathrm{YR} 5 / 8$ & $5 \mathrm{YR} 3 / 2$ & silt clay & sticky & very plastic \\
\hline SCT5 & 2a.166 & 166 & 135-145 & & Loose orange subsoil with patches of grey & none & $10 \mathrm{YR} 7 / 1$ & $7.5 \mathrm{YR} 5 / 8$ & & loam & slightly sticky & slightly plastic \\
\hline SCT5 & 2a.172 & 172 & 166-172 & & Orange subsoil with few nodules & none & 7.5 YR $5 / 8$ & & & loam & slightly sticky & slightly plastic \\
\hline SCT5 & 2a.190 & 190 & 172-177 & & Loose orange $\mathrm{C}$ horizon & none & $7.5 \mathrm{YR} 5 / 8$ & & & sandy loam & slightly sticky & not plastic \\
\hline & $3 a .30$ & & & Site off Backranch Rd, $\sim 10$ meter south of temsiometers & & & $10 \mathrm{YR} 3 / 3$ & & & & & \\
\hline 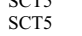 & 3a.45 & 45 & $\begin{array}{l}0-10 \\
30-36\end{array}$ & Sile orr Backranch Ka, $\sim$ IUmeter soun or temsiometers & Dark brown subsoil with nodules, clay films(?), roots & $\begin{array}{l}\text { very nime, ine } \\
\text { very fine }\end{array}$ & $10 \mathrm{YR} 2 / 3$ & & & silt loam & $\begin{array}{l}\text { Signity sticky } \\
\text { slightly sticky }\end{array}$ & $\begin{array}{l}\text { signity plastic } \\
\text { not plastic }\end{array}$ \\
\hline SCT5 & 3a. 60 & 60 & $45-53$ & & Brown subsoil with thin clay films, roots & very fine & 10YR $4 / 4$ & & & silt clay loam & $\begin{array}{l}\text { Mngmicky } \\
\text { stick }\end{array}$ & plastic \\
\hline SCT5 & $3 a .81$ & 81 & $60-65$ & & Brown, orange, red subsoil with thin clay films, roots & very fine & $10 \mathrm{YR} 5 / 4$ & $2.5 \mathrm{YR} 3 / 6$ & & silt clay loam & sticky & plastic \\
\hline SCT5 & $3 a .108$ & 108 & $81-85$ & & Grey, orange, red subsoil with thin clay films & very fine & $10 \mathrm{YR} 5 / 1$ & $2.5 \mathrm{YR} 3 / 6$ & $10 \mathrm{YR} 5 / 8$ & silt clay loam & sticky & plastic \\
\hline SCT5 & 3a. 139 & 139 & $108-111$ & & Grey, orange, red subsoil with thin clay films & very fine & $10 \mathrm{YR} 5 / 1$ & $2.5 \mathrm{YR} 3 / 6$ & $10 \mathrm{YR} 5 / 8$ & clay & very sticky & very plastic \\
\hline SCT5 & $3 a .155$ & 155 & $139-146$ & & Grainy grey, orange subsoil & none & $7.5 \mathrm{YR} 5 / 8$ & $10 \mathrm{YR} 7 / 1$ & $10 \mathrm{YR} 4 / 1$ & loam & slightly sticky & slightly plastic \\
\hline SCT5 & 3a.186 & 186 & $155-166$ & & Loose orange subsoil & none & $10 \mathrm{YR} 6 / 8$ & $10 \mathrm{YR} 7 / 1$ & & loam & slightly sticky & slightly plastic \\
\hline SCT5 & 3a.199 & 199 & 186-193 & & Loose orange $\mathrm{C}$ horizon & none & 10YR6/8 & $10 \mathrm{YR} 7 / 1$ & & sandy loam & not sticky & not plastic \\
\hline
\end{tabular}




\begin{tabular}{|c|c|c|c|c|c|c|c|c|c|c|}
\hline \multirow{3}{*}{$\begin{array}{l}\text { Terrace } \\
\text { Basal Depth (cm) }\end{array}$} & \multirow[b]{2}{*}{ SCT1-1a } & \multicolumn{9}{|c|}{ Quantified soil field properties } \\
\hline & & & & & & & & & & \\
\hline & 20 & 35 & 47 & 58 & 67 & 90 & 112 & 121 & 160 & 187 \\
\hline & 20 & 15 & 12 & 11 & 9 & 23 & 22 & 9 & 39 & 27 \\
\hline Texture & 50 & 50 & 0 & 50 & 60 & 50 & 20 & 50 & 50 & 10 \\
\hline Rubification & 0 & 20 & 40 & 40 & 60 & 70 & 70 & 70 & 40 & 40 \\
\hline Melanization & 20 & 40 & 60 & 60 & 50 & 30 & - & - & - & - \\
\hline \multirow[t]{2}{*}{$\mathrm{pH}$} & - & - & - & - & - & - & - & - & - & - \\
\hline & & \multicolumn{9}{|c|}{ Normalized Data } \\
\hline Texture & 0.56 & 0.56 & 0.00 & 0.56 & 0.67 & 0.56 & 0.22 & 0.56 & 0.56 & 0.11 \\
\hline Rubification & 0.00 & 0.11 & 0.21 & 0.21 & 0.32 & 0.37 & 0.37 & 0.37 & 0.21 & 0.21 \\
\hline Melanization & 0.29 & 0.57 & 0.86 & 0.86 & 0.71 & 0.43 & - & - & - & - \\
\hline \multirow[t]{2}{*}{$\mathrm{pH}$} & - & - & - & - & - & - & - & - & - & - \\
\hline & & \multicolumn{9}{|c|}{ Index Results } \\
\hline Sum normalized properites & 0.84 & 1.23 & 1.07 & 1.62 & 1.70 & 1.35 & 0.59 & 0.92 & 0.77 & 0.32 \\
\hline Horizon Index $(\mathrm{n}=3)$ & 0.28 & 0.41 & 0.36 & 0.54 & 0.57 & 0.45 & 0.30 & 0.46 & 0.38 & 0.16 \\
\hline Index-cm of development & 5.61 & 6.16 & 4.27 & 5.95 & 5.09 & 10.37 & 6.50 & 4.16 & 14.94 & 4.34 \\
\hline \multirow[t]{2}{*}{ Index-cm of soil profile } & 68.44 & & & Averag & index / & & 0.35 & & & \\
\hline & & \multicolumn{9}{|c|}{ Quantified soil field properties } \\
\hline Terrace & $S C T 1-2 a$ & & & & & & & & & \\
\hline Basal Depth (cm) & 10 & 19 & 45 & 50 & 74 & 92 & 104 & 118 & 142 & 172 \\
\hline Thickness $(\mathrm{cm})$ & 10 & 9 & 26 & 5 & 24 & 18 & 12 & 14 & 24 & 30 \\
\hline Texture & 60 & 60 & 40 & 20 & 120 & 120 & 100 & 30 & 20 & 20 \\
\hline Rubification & 0 & 20 & 0 & 20 & 20 & 20 & 70 & 80 & 80 & 40 \\
\hline Melanization & 40 & 80 & 60 & 40 & 40 & 60 & - & - & - & - \\
\hline \multirow[t]{2}{*}{$\mathrm{pH}$} & - & - & - & - & - & - & - & - & - & - \\
\hline & & \multicolumn{9}{|c|}{ Normalized Data } \\
\hline Texture & 0.67 & 0.67 & 0.44 & 0.22 & 1.33 & 1.33 & 1.11 & 0.33 & 0.22 & 0.22 \\
\hline Rubification & 0.00 & 0.11 & 0.00 & 0.11 & 0.11 & 0.11 & 0.37 & 0.42 & 0.42 & 0.21 \\
\hline Melanization & 0.57 & 1.14 & 0.86 & 0.57 & 0.57 & 0.86 & - & - & - & - \\
\hline \multirow[t]{2}{*}{$\mathrm{pH}$} & - & - & - & - & - & - & - & - & - & - \\
\hline & & \multicolumn{9}{|c|}{ Index Results } \\
\hline Sum normalized properites & 1.24 & 1.91 & 1.30 & 0.90 & 2.01 & 2.30 & 1.48 & 0.75 & 0.64 & 0.43 \\
\hline Horizon Index $(\mathrm{n}=3)$ & 0.41 & 0.64 & 0.43 & 0.30 & 0.67 & 0.77 & 0.74 & 0.38 & 0.32 & 0.22 \\
\hline Index-cm of development & 4.13 & 5.74 & 11.28 & 1.50 & 16.08 & 13.77 & 8.88 & 5.28 & 7.72 & 6.49 \\
\hline \multirow[t]{2}{*}{ Index-cm of soil profile } & 85.94 & & & Averag & index / & & 0.43 & & & \\
\hline & & \multicolumn{9}{|c|}{ Quantified soil field properties } \\
\hline Terrace & SCT2-1a & & & & & & & & & \\
\hline Basal Depth (cm) & 23 & 45 & 54 & 84 & 112 & 139 & 152 & 171 & 192 & 199 \\
\hline Thickness $(\mathrm{cm})$ & 23 & 22 & 9 & 30 & 28 & 27 & 13 & 19 & 21 & 7 \\
\hline Texture & 50 & 60 & 70 & 90 & 100 & 90 & 80 & 80 & 80 & 50 \\
\hline Rubification & 20 & 80 & 80 & 80 & 70 & 70 & 120 & 100 & 120 & 120 \\
\hline Melanization & 60 & 60 & 40 & 40 & - & - & - & - & - & - \\
\hline \multirow[t]{2}{*}{$\mathrm{pH}$} & - & - & - & - & - & - & - & - & - & - \\
\hline & & \multicolumn{9}{|c|}{ Normalized Data } \\
\hline Texture & 0.56 & 0.67 & 0.78 & 1.00 & 1.11 & 1.00 & 0.89 & 0.89 & 0.89 & 0.56 \\
\hline Rubification & 0.11 & 0.42 & 0.42 & 0.42 & 0.37 & 0.37 & 0.63 & 0.53 & 0.63 & 0.63 \\
\hline Melanization & 0.86 & 0.86 & 0.57 & 0.57 & - & - & - & - & - & - \\
\hline $\mathrm{pH}$ & - & - & - & - & - & - & - & - & - & - \\
\hline & & & & & & dex Res & & & & \\
\hline Sum normalized properites & 1.52 & 1.94 & 1.77 & 1.99 & 1.48 & 1.37 & 1.52 & 1.42 & 1.52 & 1.19 \\
\hline Horizon Index $(\mathrm{n}=3)$ & 0.51 & 0.65 & 0.59 & 0.66 & 0.74 & 0.68 & 0.76 & 0.71 & 0.76 & 0.59 \\
\hline Index-cm of development & 11.64 & 14.26 & 5.31 & 19.92 & 20.71 & 18.47 & 9.88 & 13.44 & 15.96 & 4.15 \\
\hline Index-cm of soil profile & 133.77 & & & Averag & index / & & 0.67 & & & \\
\hline
\end{tabular}




\begin{tabular}{|c|c|c|c|c|c|c|c|c|c|c|c|}
\hline \multirow{3}{*}{$\begin{array}{l}\text { Terrace } \\
\text { Basal Denth }(\mathrm{cm})\end{array}$} & \multicolumn{11}{|c|}{ Quantified soil field properties } \\
\hline & \multicolumn{3}{|l|}{$S C T 2-2 a$} & & \multirow[b]{2}{*}{86} & \multirow{3}{*}{$\begin{array}{c}103 \\
17\end{array}$} & \multirow{3}{*}{$\begin{array}{c}115 \\
12\end{array}$} & \multirow{3}{*}{$\begin{array}{c}145 \\
30\end{array}$} & \multirow[b]{2}{*}{181} & \multirow[b]{2}{*}{191} & \multirow[b]{2}{*}{200} \\
\hline & 13 & 34 & 51 & 66 & & & & & & & \\
\hline Thickness $(\mathrm{cm})$ & 13 & 21 & 17 & 15 & 20 & & & & 36 & 10 & 9 \\
\hline Texture & 50 & 50 & 50 & 70 & 80 & 120 & 120 & 120 & 120 & 80 & 80 \\
\hline Rubification & 20 & 40 & 40 & 80 & 110 & 80 & 80 & 90 & 90 & 50 & 80 \\
\hline Melanization & 40 & 60 & 60 & 40 & 40 & - & - & - & - & - & - \\
\hline \multirow[t]{2}{*}{$\mathrm{pH}$} & - & - & - & - & - & - & - & - & - & - & - \\
\hline & \multicolumn{11}{|c|}{ Normalized Data } \\
\hline Texture & 0.56 & 0.56 & 0.56 & 0.78 & 0.89 & 1.33 & 1.33 & 1.33 & 1.33 & 0.89 & 0.89 \\
\hline Rubification & 0.11 & 0.21 & 0.21 & 0.42 & 0.58 & 0.42 & 0.42 & 0.47 & 0.47 & 0.26 & 0.42 \\
\hline Melanization & 0.57 & 0.86 & 0.86 & 0.57 & 0.57 & - & - & - & - & - & - \\
\hline \multirow[t]{2}{*}{$\mathrm{pH}$} & - & - & - & - & - & - & - & - & - & - & - \\
\hline & \multicolumn{11}{|c|}{ Index Results } \\
\hline Sum normalized properites & 1.23 & 1.62 & 1.62 & 1.77 & 2.04 & 1.75 & 1.75 & 1.81 & 1.81 & 1.15 & 1.31 \\
\hline Horizon Index $(n=3)$ & 0.41 & 0.54 & 0.54 & 0.59 & 0.68 & 0.88 & 0.88 & 0.90 & 0.90 & 0.58 & 0.65 \\
\hline Index-cm of development & 5.34 & 11.36 & 9.20 & 8.85 & 13.60 & 14.91 & 10.53 & 27.11 & 32.53 & 5.76 & 5.89 \\
\hline \multirow[t]{2}{*}{ Index-cm of soil profile } & 145.07 & & & Averag & index / & & 0.73 & & & & \\
\hline & & & & & antified & soil fiel & proper & & & & \\
\hline Terrace & $S C T 2-1$ & & & & & & & & & & \\
\hline Basal Depth (cm) & 5 & 13 & 39 & 50 & 68 & 88 & 100 & & & & \\
\hline Thickness $(\mathrm{cm})$ & 5 & 8 & 26 & 11 & 18 & 20 & 12 & & & & \\
\hline Texture & 30 & 30 & 50 & 80 & 80 & 70 & 90 & & & & \\
\hline Rubification & 20 & 20 & 0 & 80 & 80 & 80 & 80 & & & & \\
\hline Melanization & 80 & 80 & 80 & 60 & 60 & 50 & 50 & & & & \\
\hline $\mathrm{pH}$ & 1.2 & 1.2 & 1.7 & 0.2 & 0.2 & 0.2 & 0.2 & & & & \\
\hline & & & & & Nor & nalized & Data & & & & \\
\hline Texture & 0.33 & 0.33 & 0.56 & 0.89 & 0.89 & 0.78 & 1.00 & & & & \\
\hline Rubification & 0.11 & 0.11 & 0.00 & 0.42 & 0.42 & 0.42 & 0.42 & & & & \\
\hline Melanization & 1.14 & 1.14 & 1.14 & 0.86 & 0.86 & 0.71 & 0.71 & & & & \\
\hline $\mathrm{pH}$ & 0.3 & 0.3 & 0.5 & 0.1 & 0.1 & 0.1 & 0.1 & & & & \\
\hline & & & & & & lex Res & & & & & \\
\hline Sum normalized properites & 1.92 & 1.92 & 2.18 & 2.22 & 2.22 & 1.97 & 2.19 & & & & \\
\hline Horizon Index $(n=4)$ & 0.48 & 0.48 & 0.55 & 0.56 & 0.56 & 0.49 & 0.55 & & & & \\
\hline Index-cm of development & 2.41 & 3.85 & 14.20 & 6.12 & 10.01 & 9.85 & 6.58 & & & & \\
\hline Index-cm of soil profile & 53.01 & & & Averag & index / & & 0.53 & & & & \\
\hline & & & & & antified & soil fiel & proper & & & & \\
\hline Terrace & $S C T 2-2$ & & & & & & & & & & \\
\hline Basal Depth (cm) & 12 & 22 & 44 & 65 & 85 & 100 & & & & & \\
\hline Thickness $(\mathrm{cm})$ & 12 & 10 & 22 & 21 & 20 & 15 & & & & & \\
\hline Texture & 50 & 50 & 50 & 70 & 80 & 40 & & & & & \\
\hline Rubification & 10 & 0 & 30 & 50 & 60 & 80 & & & & & \\
\hline Melanization & 40 & 40 & 40 & 60 & 40 & 50 & & & & & \\
\hline $\mathrm{pH}$ & - & - & - & - & - & - & & & & & \\
\hline & & & & & Nor & nalized & Data & & & & \\
\hline Texture & 0.56 & 0.56 & 0.56 & 0.78 & 0.89 & 0.44 & & & & & \\
\hline Rubification & 0.08 & 0.00 & 0.25 & 0.42 & 0.50 & 0.67 & & & & & \\
\hline Melanization & 0.33 & 0.33 & 0.33 & 0.50 & 0.33 & 0.42 & & & & & \\
\hline $\mathrm{pH}$ & - & - & - & - & - & - & & & & & \\
\hline & & & & & & lex Res & & & & & \\
\hline Sum normalized properites & 0.97 & 0.89 & 1.14 & 1.69 & 1.72 & 1.53 & & & & & \\
\hline Horizon Index $(n=3)$ & 0.32 & 0.30 & 0.38 & 0.56 & 0.57 & 0.51 & & & & & \\
\hline Index-cm of development & 3.89 & 2.96 & 8.35 & 11.86 & 11.48 & 7.64 & & & & & \\
\hline Index-cm of soil profile & 46.19 & & & Averag & index / & & 0.46 & & & & \\
\hline
\end{tabular}




\begin{tabular}{|c|c|c|c|c|c|c|c|c|c|c|}
\hline \multirow{3}{*}{$\begin{array}{l}\text { Terrace } \\
\text { Basal Depth (cm) }\end{array}$} & \multirow[b]{2}{*}{ SCT3-1a } & \multicolumn{9}{|c|}{ Quantified soil field properties } \\
\hline & & & & & & & & & & \\
\hline & 26 & 45 & 56 & 70 & 84 & 110 & 145 & 150 & 160 & 184 \\
\hline Thickness $(\mathrm{cm})$ & 26 & 19 & 11 & 14 & 14 & 26 & 35 & 5 & 10 & 24 \\
\hline Texture & 60 & 60 & 80 & 80 & 80 & 110 & 110 & 80 & 90 & 80 \\
\hline Rubification & 20 & 80 & 120 & 40 & 90 & 80 & 100 & 110 & 80 & 80 \\
\hline Melanization & 40 & 40 & 40 & 40 & 50 & - & - & - & - & - \\
\hline \multirow[t]{2}{*}{$\mathrm{pH}$} & - & - & - & - & - & - & - & - & - & - \\
\hline & & \multicolumn{9}{|c|}{ Normalized Data } \\
\hline Texture & 0.67 & 0.67 & 0.89 & 0.89 & 0.89 & 1.22 & 1.22 & 0.89 & 1.00 & 0.89 \\
\hline Rubification & 0.17 & 0.67 & 1.00 & 0.33 & 0.75 & 0.67 & 0.83 & 0.92 & 0.67 & 0.67 \\
\hline Melanization & 0.33 & 0.33 & 0.33 & 0.33 & 0.42 & - & - & - & - & - \\
\hline \multirow[t]{2}{*}{$\mathrm{pH}$} & - & - & - & - & - & - & - & - & - & - \\
\hline & & \multicolumn{9}{|c|}{ Index Results } \\
\hline Sum normalized properites & 1.17 & 1.67 & 2.22 & 1.56 & 2.06 & 1.89 & 2.06 & 1.81 & 1.67 & 1.56 \\
\hline Horizon Index $(n=3)$ & 0.39 & 0.56 & 0.74 & 0.52 & 0.69 & 0.94 & 1.03 & 0.90 & 0.83 & 0.78 \\
\hline Index-cm of development & 10.11 & 10.56 & 8.15 & 7.26 & 9.59 & 24.56 & 35.97 & 4.51 & 8.33 & 18.67 \\
\hline \multirow[t]{2}{*}{ Index-cm of soil profile } & 151.49 & & & Averag & index / & & 0.76 & & & \\
\hline & & \multicolumn{9}{|c|}{ Quantified soil field properties } \\
\hline Terrace & SCT3-2a & & & & & & & & & \\
\hline Basal Depth (cm) & 32 & 40 & 57 & 78 & 90 & 107 & 117 & 140 & 150 & 190 \\
\hline Thickness $(\mathrm{cm})$ & 32 & 8 & 17 & 21 & 12 & 17 & 10 & 23 & 10 & 40 \\
\hline Texture & 60 & 60 & 60 & 90 & 100 & 120 & 120 & 120 & 120 & 80 \\
\hline Rubification & 40 & 40 & 80 & 80 & 70 & 90 & 110 & 80 & 80 & 120 \\
\hline Melanization & 60 & 60 & 40 & 30 & 60 & - & - & - & - & - \\
\hline \multirow[t]{2}{*}{$\mathrm{pH}$} & - & - & - & - & - & - & - & - & - & - \\
\hline & & \multicolumn{9}{|c|}{ Normalized Data } \\
\hline Texture & 0.67 & 0.67 & 0.67 & 1.00 & 1.11 & 1.33 & 1.33 & 1.33 & 1.33 & 0.89 \\
\hline Rubification & 0.33 & 0.33 & 0.67 & 0.67 & 0.58 & 0.75 & 0.92 & 0.67 & 0.67 & 1.00 \\
\hline Melanization & 0.50 & 0.50 & 0.33 & 0.25 & 0.50 & - & - & - & - & - \\
\hline \multirow[t]{2}{*}{$\mathrm{pH}$} & - & - & - & - & - & - & - & - & - & - \\
\hline & & \multicolumn{9}{|c|}{ Index Results } \\
\hline Sum normalized properites & 1.50 & 1.50 & 1.67 & 1.92 & 2.19 & 2.08 & 2.25 & 2.00 & 2.00 & 1.89 \\
\hline Horizon Index $(n=3)$ & 0.50 & 0.50 & 0.56 & 0.64 & 0.73 & 1.04 & 1.13 & 1.00 & 1.00 & 0.94 \\
\hline Index-cm of development & 16.00 & 4.00 & 9.44 & 13.42 & 8.78 & 17.71 & 11.25 & 23.00 & 10.00 & 37.78 \\
\hline \multirow[t]{2}{*}{ Index-cm of soil profile } & 160.94 & & & Averag & index/ & & 0.79 & & & \\
\hline & & \multicolumn{9}{|c|}{ Quantified soil field properties } \\
\hline Terrace & SCT3-3a & & & & & & & & & \\
\hline Basal Depth (cm) & 17 & 28 & 40 & 63 & 72 & 84 & 95 & 110 & 128 & 150 \\
\hline Thickness $(\mathrm{cm})$ & 17 & 11 & 12 & 23 & 9 & 12 & 11 & 15 & 18 & 22 \\
\hline Texture & 60 & 60 & 60 & 90 & 120 & 120 & 120 & 120 & 80 & 80 \\
\hline Rubification & 40 & 120 & 80 & 90 & 90 & 80 & 80 & 80 & 90 & 90 \\
\hline Melanization & 60 & 40 & 40 & 40 & 30 & 40 & 50 & - & - & - \\
\hline \multirow[t]{2}{*}{$\mathrm{pH}$} & - & - & - & - & - & - & - & - & - & - \\
\hline & & \multicolumn{9}{|c|}{ Normalized Data } \\
\hline Texture & 0.67 & 0.67 & 0.67 & 1.00 & 1.33 & 1.33 & 1.33 & 1.33 & 0.89 & 0.89 \\
\hline Rubification & 0.33 & 1.00 & 0.67 & 0.75 & 0.75 & 0.67 & 0.67 & 0.67 & 0.75 & 0.75 \\
\hline Melanization & 0.50 & 0.33 & 0.33 & 0.33 & 0.25 & 0.33 & 0.42 & - & - & - \\
\hline $\mathrm{pH}$ & - & - & - & - & - & - & - & - & - & - \\
\hline & & & & & & dex Res & & & & \\
\hline Sum normalized properites & 1.50 & 2.00 & 1.67 & 2.08 & 2.33 & 2.33 & 2.42 & 2.00 & 1.64 & 1.64 \\
\hline Horizon Index $(\mathrm{n}=3)$ & 0.50 & 0.67 & 0.56 & 0.69 & 0.78 & 0.78 & 0.81 & 1.00 & 0.82 & 0.82 \\
\hline Index-cm of development & 8.50 & 7.33 & 6.67 & 15.97 & 7.00 & 9.33 & 8.86 & 15.00 & 14.75 & 18.03 \\
\hline Index-cm of soil profile & 111.44 & & & Averag & index / & & 0.74 & & & \\
\hline
\end{tabular}




\begin{tabular}{|c|c|c|c|c|c|c|c|c|c|c|}
\hline \multirow{3}{*}{$\begin{array}{l}\text { Terrace } \\
\text { Basal Denth }(\mathrm{cm})\end{array}$} & \multirow[b]{2}{*}{ SCT3-1 } & \multicolumn{9}{|c|}{ Quantified soil field properties } \\
\hline & & & & & & & & & & \\
\hline & 5 & 10 & 20 & 30 & 55 & 70 & 90 & 100 & & \\
\hline Thickness $(\mathrm{cm})$ & 5 & 5 & 10 & 10 & 25 & 15 & 20 & 10 & & \\
\hline Texture & 50 & 50 & 50 & 50 & 60 & 80 & 80 & 90 & & \\
\hline Rubification & 10 & 10 & 20 & 20 & 30 & 75 & 60 & 100 & & \\
\hline Melanization & 60 & 50 & 50 & 60 & 40 & 40 & 40 & 40 & & \\
\hline \multirow[t]{2}{*}{$\mathrm{pH}$} & 1.7 & 1.6 & 1.4 & 1.6 & 1.4 & 1.6 & 1.5 & 1.8 & & \\
\hline & & \multicolumn{9}{|c|}{ Normalized Data } \\
\hline Texture & 0.56 & 0.56 & 0.56 & 0.56 & 0.67 & 0.89 & 0.89 & 1.00 & & \\
\hline Rubification & 0.08 & 0.08 & 0.17 & 0.17 & 0.25 & 0.63 & 0.50 & 0.83 & & \\
\hline Melanization & 0.50 & 0.42 & 0.42 & 0.50 & 0.33 & 0.33 & 0.33 & 0.33 & & \\
\hline \multirow[t]{2}{*}{$\mathrm{pH}$} & 0.5 & 0.5 & 0.4 & 0.5 & 0.4 & 0.5 & 0.4 & 0.5 & & \\
\hline & & \multicolumn{9}{|c|}{ Index Results } \\
\hline Sum normalized properites & 1.62 & 1.51 & 1.54 & 1.68 & 1.65 & 2.30 & 2.15 & \multirow{4}{*}{$\begin{array}{l}2.68 \\
0.67 \\
6.70\end{array}$} & & \\
\hline Horizon Index $(n=4)$ & 0.41 & 0.38 & 0.38 & 0.42 & 0.41 & 0.58 & 0.54 & & & \\
\hline Index-cm of development & 2.03 & 1.89 & 3.85 & 4.20 & 10.31 & 8.64 & 10.75 & & & \\
\hline \multirow[t]{2}{*}{ Index-cm of soil profile } & 48.38 & & & \multicolumn{3}{|c|}{ Average index / cm } & 0.48 & & & \\
\hline & & \multicolumn{9}{|c|}{ Quantified soil field properties } \\
\hline Terrace & SCT4-1a & & & & & & & & & \\
\hline Basal Depth (cm) & 24 & 45 & 54 & 74 & 110 & 135 & 157 & 171 & 195 & 204 \\
\hline Thickness (cm) & 24 & 21 & 9 & 20 & 36 & 25 & 22 & 14 & 24 & 9 \\
\hline Texture & 40 & 40 & 40 & 80 & 80 & 100 & 110 & 90 & 90 & 90 \\
\hline Rubification & 40 & 100 & 80 & 100 & 80 & 80 & 80 & 100 & 100 & 50 \\
\hline Melanization & 60 & 60 & 60 & 70 & - & - & - & - & - & - \\
\hline \multirow[t]{2}{*}{$\mathrm{pH}$} & - & - & - & - & - & - & - & - & - & - \\
\hline & & \multicolumn{9}{|c|}{ Normalized Data } \\
\hline Texture & 0.44 & 0.44 & 0.44 & 0.89 & 0.89 & 1.11 & 1.22 & 1.00 & 1.00 & 1.00 \\
\hline Rubification & 0.33 & 0.83 & 0.67 & 0.83 & 0.67 & 0.67 & 0.67 & 0.83 & 0.83 & 0.42 \\
\hline Melanization & 0.50 & 0.50 & 0.50 & 0.58 & - & - & - & - & - & - \\
\hline \multirow[t]{2}{*}{$\mathrm{pH}$} & - & - & - & - & - & - & - & - & - & - \\
\hline & & \multicolumn{9}{|c|}{ Index Results } \\
\hline Sum normalized properites & 1.28 & 1.78 & 1.61 & 2.31 & 1.56 & 1.78 & 1.89 & 1.83 & 1.83 & 1.42 \\
\hline Horizon Index $(n=3)$ & 0.43 & 0.59 & 0.54 & 0.77 & 0.78 & 0.89 & 0.94 & 0.92 & 0.92 & 0.71 \\
\hline Index-cm of development & 10.22 & 12.44 & 4.83 & 15.37 & 28.00 & 22.22 & 20.78 & 12.83 & 22.00 & 6.38 \\
\hline \multirow[t]{2}{*}{ Index-cm of soil profile } & 155.08 & & & \multicolumn{3}{|c|}{ Average index / cm } & 0.76 & & & \\
\hline & & & & & antified & ioil fiel & proper & & & \\
\hline Terrace & SCT4-2a & & & & & & & & & \\
\hline Basal Depth (cm) & 29 & 46 & 64 & 81 & 106 & 125 & 135 & & & \\
\hline Thickness (cm) & 29 & 17 & 18 & 17 & 25 & 19 & 10 & & & \\
\hline Texture & 30 & 40 & 60 & 60 & 90 & 80 & 20 & & & \\
\hline Rubification & 40 & 80 & 80 & 120 & 80 & 80 & 150 & & & \\
\hline Melanization & 60 & 60 & 40 & 50 & - & - & - & & & \\
\hline $\mathrm{pH}$ & - & - & - & - & - & - & - & & & \\
\hline & & & & & Nor & ralized & Data & & & \\
\hline Texture & 0.33 & 0.44 & 0.67 & 0.67 & 1.00 & 0.89 & 0.22 & & & \\
\hline Rubification & 0.33 & 0.67 & 0.67 & 1.00 & 0.67 & 0.67 & 1.25 & & & \\
\hline Melanization & 0.50 & 0.50 & 0.33 & 0.42 & - & - & - & & & \\
\hline $\mathrm{pH}$ & - & - & - & - & - & - & - & & & \\
\hline & & & & & & ex Res & & & & \\
\hline Sum normalized properites & 1.17 & 1.61 & 1.67 & 2.08 & 1.67 & 1.56 & 1.47 & & & \\
\hline Horizon Index $(n=3)$ & 0.39 & 0.54 & 0.56 & 0.69 & 0.83 & 0.78 & 0.74 & & & \\
\hline Index-cm of development & 11.28 & 9.13 & 10.00 & 11.81 & 20.83 & 14.78 & 7.36 & & & \\
\hline Index-cm of soil profile & 85.19 & & & Averag & index / & & 0.63 & & & \\
\hline
\end{tabular}




\begin{tabular}{|c|c|c|c|c|c|c|c|c|c|c|c|}
\hline \multirow{3}{*}{$\begin{array}{l}\text { Terrace } \\
\text { Basal Depth }(\mathrm{cm})\end{array}$} & \multicolumn{11}{|c|}{ Quantified soil field properties } \\
\hline & \multicolumn{3}{|l|}{ SCT4-3a } & \multirow[b]{2}{*}{63} & \multirow[b]{2}{*}{88} & \multirow[b]{2}{*}{100} & \multirow{3}{*}{$\begin{array}{c}109 \\
9\end{array}$} & \multirow[b]{2}{*}{140} & \multirow[b]{2}{*}{170} & \multirow[b]{2}{*}{198} & \multirow[b]{2}{*}{206} \\
\hline & 17 & 30 & 40 & & & & & & & & \\
\hline Thickness $(\mathrm{cm})$ & 17 & 13 & 10 & 23 & 25 & 12 & & 31 & 30 & 28 & 8 \\
\hline Texture & 40 & 50 & 50 & 80 & 80 & 120 & 120 & 120 & 120 & 80 & 80 \\
\hline Rubification & 40 & 80 & 80 & 80 & 80 & 90 & 100 & 90 & 100 & 70 & 70 \\
\hline Melanization & 60 & 40 & 60 & 60 & 50 & 40 & - & - & - & - & - \\
\hline \multirow[t]{2}{*}{$\mathrm{pH}$} & - & - & - & - & - & - & - & - & - & - & - \\
\hline & \multicolumn{11}{|c|}{ Normalized Data } \\
\hline Texture & 0.44 & 0.56 & 0.56 & 0.89 & 0.89 & 1.33 & 1.33 & 1.33 & 1.33 & 0.89 & 0.89 \\
\hline Rubification & 0.33 & 0.67 & 0.67 & 0.67 & 0.67 & 0.75 & 0.83 & 0.75 & 0.83 & 0.58 & 0.58 \\
\hline Melanization & 0.50 & 0.33 & 0.50 & 0.50 & 0.42 & 0.33 & - & - & - & - & - \\
\hline \multirow[t]{2}{*}{$\mathrm{pH}$} & - & - & - & - & - & - & - & - & - & - & - \\
\hline & \multicolumn{11}{|c|}{ Index Results } \\
\hline Sum normalized properites & 1.28 & 1.56 & 1.72 & 2.06 & 1.97 & 2.42 & 2.17 & 2.08 & 2.17 & 1.47 & 1.47 \\
\hline Horizon Index $(\mathrm{n}=3)$ & 0.43 & 0.52 & 0.57 & 0.69 & 0.66 & 0.81 & 1.08 & 1.04 & 1.08 & 0.74 & 0.74 \\
\hline Index-cm of development & 7.24 & 6.74 & 5.74 & 15.76 & 16.44 & 9.67 & 9.75 & 32.29 & 32.50 & 20.61 & 5.89 \\
\hline \multirow[t]{2}{*}{ Index-cm of soil profile } & 162.63 & & & Averag & index / & & 0.79 & & & & \\
\hline & & & & & Iantified & soil fiel & prope & & & & \\
\hline Terrace & SCT4-1 & & & & & & & & & & \\
\hline Basal Depth (cm) & 10 & 20 & 28 & 40 & 60 & 80 & 100 & & & & \\
\hline Thickness $(\mathrm{cm})$ & 10 & 10 & 8 & 12 & 20 & 20 & 20 & & & & \\
\hline Texture & 40 & 50 & 70 & 50 & 60 & 80 & 80 & & & & \\
\hline Rubification & 20 & 55 & 100 & 100 & 130 & 100 & 60 & & & & \\
\hline Melanization & 60 & 60 & 50 & 50 & 40 & 30 & 20 & & & & \\
\hline $\mathrm{pH}$ & 1.2 & 1.2 & 1.0 & 1.0 & 0.6 & 0.8 & 0.2 & & & & \\
\hline & & & & & Nor & nalized & ata & & & & \\
\hline Texture & 0.44 & 0.56 & 0.78 & 0.56 & 0.67 & 0.89 & 0.89 & & & & \\
\hline Rubification & 0.17 & 0.46 & 0.83 & 0.83 & 1.08 & 0.83 & 0.50 & & & & \\
\hline Melanization & 0.50 & 0.50 & 0.42 & 0.42 & 0.33 & 0.25 & 0.17 & & & & \\
\hline $\mathrm{pH}$ & 0.3 & 0.3 & 0.3 & 0.3 & 0.2 & 0.2 & 0.1 & & & & \\
\hline & & & & & & dex Res & & & & & \\
\hline Sum normalized properites & 1.45 & 1.86 & 2.31 & 2.09 & 2.25 & 2.20 & 1.61 & & & & \\
\hline Horizon Index $(\mathrm{n}=3)$ & 0.36 & 0.46 & 0.58 & 0.52 & 0.56 & 0.55 & 0.40 & & & & \\
\hline Index-cm of development & 3.63 & 4.64 & 4.63 & 6.27 & 11.27 & 11.00 & 8.06 & & & & \\
\hline Index-cm of soil profile & 49.52 & & & Averag & index / & & 0.50 & & & & \\
\hline & & & & & Iantified & soil fiel & prope & & & & \\
\hline Terrace & SCT5-1 & & & & & & & & & & \\
\hline Basal Depth (cm) & 10 & 20 & 37 & 55 & 80 & 100 & & & & & \\
\hline Thickness $(\mathrm{cm})$ & 10 & 10 & 17 & 18 & 25 & 20 & & & & & \\
\hline Texture & 50 & 50 & 50 & 80 & 80 & 80 & & & & & \\
\hline Rubification & 0 & 20 & 35 & 60 & 40 & 50 & & & & & \\
\hline Melanization & 40 & 60 & 75 & 50 & 50 & 50 & & & & & \\
\hline $\mathrm{pH}$ & 1.0 & 1.0 & 0.6 & 1.4 & 1.6 & 2.0 & & & & & \\
\hline & & & & & Nor & nalized & ata & & & & \\
\hline Texture & 0.56 & 0.56 & 0.56 & 0.89 & 0.89 & 0.89 & & & & & \\
\hline Rubification & 0.00 & 0.17 & 0.29 & 0.50 & 0.33 & 0.42 & & & & & \\
\hline Melanization & 0.33 & 0.50 & 0.63 & 0.42 & 0.42 & 0.42 & & & & & \\
\hline $\mathrm{pH}$ & 0.3 & 0.3 & 0.2 & 0.4 & 0.5 & 0.6 & & & & & \\
\hline & & & & & & dex Res & & & & & \\
\hline Sum normalized properites & 1.17 & 1.51 & 1.64 & 2.21 & 2.10 & 2.29 & & & & & \\
\hline Horizon Index $(n=4)$ & 0.29 & 0.38 & 0.41 & 0.55 & 0.52 & 0.57 & & & & & \\
\hline Index-cm of development & 2.94 & 3.77 & 6.99 & 9.93 & 13.10 & 11.47 & & & & & \\
\hline Index-cm of soil profile & 48.19 & & & Averag & index / & & 0.48 & & & & \\
\hline
\end{tabular}




\begin{tabular}{|c|c|c|c|c|c|c|c|c|c|}
\hline \multirow{3}{*}{ Terrace } & \multirow[b]{2}{*}{ SCT5-1a } & & \multicolumn{7}{|c|}{ Quantified soil field properties } \\
\hline & & & & & & & & & \\
\hline & 24 & 45 & 60 & 87 & 114 & 133 & 149 & 177 & 193 \\
\hline Thickness (cm) & 24 & 21 & 15 & 27 & 27 & 19 & 16 & 28 & 16 \\
\hline Texture & 50 & 50 & 90 & 100 & 100 & 90 & 90 & 60 & 20 \\
\hline Rubification & 20 & 20 & 130 & 90 & 100 & 100 & 80 & 70 & 70 \\
\hline Melanization & 80 & 60 & 50 & 40 & - & - & - & - & - \\
\hline \multirow[t]{2}{*}{$\mathrm{pH}$} & - & - & - & - & - & - & - & - & - \\
\hline & & & \multicolumn{7}{|c|}{ Normalized Data } \\
\hline Texture & 0.56 & 0.56 & 1.00 & 1.11 & 1.11 & 1.00 & 1.00 & 0.67 & 0.22 \\
\hline Rubification & 0.17 & 0.17 & 1.08 & 0.75 & 0.83 & 0.83 & 0.67 & 0.58 & 0.58 \\
\hline Melanization & 0.67 & 0.50 & 0.42 & 0.33 & - & - & - & - & - \\
\hline \multirow[t]{2}{*}{$\mathrm{pH}$} & - & - & - & - & - & - & - & - & - \\
\hline & & & \multicolumn{7}{|c|}{ Index Results } \\
\hline Sum normalized properites & 1.39 & 1.22 & 2.50 & 2.19 & 1.94 & 1.83 & 1.67 & 1.25 & 0.81 \\
\hline Horizon Index $(n=3)$ & 0.46 & 0.41 & 0.83 & 0.73 & 0.97 & 0.92 & 0.83 & 0.63 & 0.40 \\
\hline Index-cm of development & 11.11 & 8.56 & 12.50 & 19.75 & 26.25 & 17.42 & 13.33 & 17.50 & 6.44 \\
\hline \multirow[t]{2}{*}{ Index-cm of soil profile } & 135.68 & & & Average & index / & & 0.68 & & \\
\hline & & & \multicolumn{7}{|c|}{ Quantified soil field properties } \\
\hline Terrace & SCT5-2a & & & & & & & & \\
\hline Basal Depth (cm) & 16 & 34 & 55 & 66 & 85 & 111 & 135 & 166 & 172 \\
\hline Thickness (cm) & 16 & 18 & 21 & 11 & 19 & 26 & 24 & 31 & 6 \\
\hline Texture & 60 & 50 & 70 & 70 & 70 & 110 & 110 & 50 & 50 \\
\hline Rubification & 20 & 40 & 80 & 60 & 80 & 80 & 80 & 80 & 140 \\
\hline Melanization & 60 & 60 & 60 & 60 & 60 & - & - & - & - \\
\hline \multirow[t]{2}{*}{$\mathrm{pH}$} & - & - & - & - & - & - & - & - & - \\
\hline & & & \multicolumn{7}{|c|}{ Normalized Data } \\
\hline Texture & 0.67 & 0.56 & 0.78 & 0.78 & 0.78 & 1.22 & 1.22 & 0.56 & 0.56 \\
\hline Rubification & 0.17 & 0.33 & 0.67 & 0.50 & 0.67 & 0.67 & 0.67 & 0.67 & 1.17 \\
\hline Melanization & 0.50 & 0.50 & 0.50 & 0.50 & 0.50 & - & - & - & - \\
\hline \multirow[t]{2}{*}{$\mathrm{pH}$} & - & - & - & - & - & - & - & - & - \\
\hline & & & \multicolumn{7}{|c|}{ Index Results } \\
\hline Sum normalized properites & 1.33 & 1.39 & 1.94 & 1.78 & 1.94 & 1.89 & 1.89 & 1.22 & 1.72 \\
\hline Horizon Index $(n=3)$ & 0.44 & 0.46 & 0.65 & 0.59 & 0.65 & 0.94 & 0.94 & 0.61 & 0.86 \\
\hline Index-cm of development & 7.11 & 8.33 & 13.61 & 6.52 & 12.31 & 24.56 & 22.67 & 18.94 & 5.17 \\
\hline \multirow[t]{2}{*}{ Index-cm of soil profile } & 132.72 & & & Averag & index / & & 0.70 & & \\
\hline & & & \multicolumn{7}{|c|}{ Quantified soil field properties } \\
\hline Terrace & SCT5-3a & & & & & & & & \\
\hline Basal Depth (cm) & 30 & 45 & 60 & 81 & 108 & 139 & 155 & 186 & 199 \\
\hline Thickness $(\mathrm{cm})$ & 30 & 15 & 15 & 21 & 27 & 31 & 16 & 31 & 13 \\
\hline Texture & 50 & 50 & 90 & 90 & 90 & 120 & 50 & 50 & 20 \\
\hline Rubification & 20 & 20 & 40 & 90 & 100 & 100 & 80 & 70 & 70 \\
\hline Melanization & 80 & 100 & 60 & 60 & - & - & - & - & - \\
\hline \multirow[t]{2}{*}{$\mathrm{pH}$} & - & - & - & - & - & - & - & - & - \\
\hline & & & \multicolumn{7}{|c|}{ Normalized Data } \\
\hline Texture & 0.56 & 0.56 & 1.00 & 1.00 & 1.00 & 1.33 & 0.56 & 0.56 & 0.22 \\
\hline Rubification & 0.17 & 0.17 & 0.33 & 0.75 & 0.83 & 0.83 & 0.67 & 0.58 & 0.58 \\
\hline Melanization & 0.67 & 0.83 & 0.50 & 0.50 & - & - & - & - & - \\
\hline $\mathrm{pH}$ & - & - & - & - & - & - & - & - & - \\
\hline & & & & & & ex Res & & & \\
\hline Sum normalized properites & 1.39 & 1.56 & 1.83 & 2.25 & 1.83 & 2.17 & 1.22 & 1.14 & 0.81 \\
\hline Horizon Index $(\mathrm{n}=3)$ & 0.46 & 0.52 & 0.61 & 0.75 & 0.92 & 1.08 & 0.61 & 0.57 & 0.40 \\
\hline Index-cm of development & 13.89 & 7.78 & 9.17 & 15.75 & 24.75 & 33.58 & 9.78 & 17.65 & 5.24 \\
\hline Index-cm of soil profile & 137.58 & & & Averag & index / & & 0.69 & & \\
\hline
\end{tabular}

